\title{
Innovative Strategy to Improve Precision and to Save Power of a Real-Time Control Process Using an Online Adaptive Fuzzy Logic Controller
}

\author{
R. Lasri, ${ }^{1}$ I. Rojas, ${ }^{1}$ H. Pomares, ${ }^{1}$ and O. Valenzuela ${ }^{2}$ \\ ${ }^{1}$ Department of Computer Architecture \& Computer Technology, University of Granada, 18071 Granada, Spain \\ ${ }^{2}$ Department of Applied Mathematics, University of Granada, 18071 Granada, Spain \\ Correspondence should be addressed to R. Lasri; rlasri@ugr.es
}

Received 5 March 2013; Accepted 21 May 2013

Academic Editor: M. Onder Efe

Copyright (C) 2013 R. Lasri et al. This is an open access article distributed under the Creative Commons Attribution License, which permits unrestricted use, distribution, and reproduction in any medium, provided the original work is properly cited.

\begin{abstract}
The main objective of this paper is to prove the great advantage that brings our novel approach to the intelligent control area. A set of various types of intelligent controllers have been designed to control the temperature of a room in a real-time control process in order to compare the obtained results with each other. Through a training board that allows us to control the temperature, all the used algorithms should present their best performances in this control process; therefore, our self-organized and online adaptive fuzzy logic controller (FLC) will be required to present great improvements in the control task and a real high control performance. Simulation results can show clearly that the new approach presented and tested in this work is very efficient. Thus, our adaptive and self-organizing FLC presents the best accuracy compared with the remaining used controllers, and, besides that, it can guarantee an important reduction of the power consumption during the control process.
\end{abstract}

\section{Introduction}

An intelligent control system typically consists of two parts: the first is the "knowledge base" which presents the necessary knowledge to control the plant, and the second is an "inference engine" which processes the knowledge through reasoning, possibly using a new set of data to obtain the decision. Therefore, both, the representation and the processing, are critical in the "intelligent control." Our study uses intelligent control techniques based on fuzzy logic and PID controller structures. Several structures of controllers have been tested in this control process to demonstrate the profitability of our adaptive fuzzy controller.

During the past 30 years numerous studies have presented several examples of adaptive controllers. In the 1970 Mamdani and his student Assilian invented an adaptive controller [1] with the objective to determine the responsible rule for the undesirable state of the plant and replace it with the appropriate value. These steps have initialized a new policy of adaptive fuzzy logic controllers called Self-Organizing Control (SOC) system [2]. In most SOC approaches, this dependence is expressed using only the monotonicity sign of the plant, that is, the sign of the relation between the plant output and the controller output, since the knowledge of the Jacobian matrix is hardly ever available. SOCs have proved to be successful in "simple" applications since only the rule consequents have to be tuned. In highly nonlinear plants, SOCs are only capable of a coarse tuning of the controller parameters; therefore, more sophisticated fuzzy controllers have to be constructed [3-20]. For example, in [11], a hybrid adaptive fuzzy control is suggested where the nonlinear system is controlled by a state feedback controller and an adaptive fuzzy controller. The main advantage of the proposed approach is that the adaptive fuzzy control law can tune the robust gain of the slidingmode controller so as to cope with the uncertainties and modeling error of the nonlinear robotic system. In [3] a fuzzy controller is constructed from a fuzzy feedback linearization controller, whose parameters are adjusted indirectly from the estimates of the plant parameters, providing asymptotic tracking of a reference signal for a system with uncertainty or slowly time-varying parameters. The main drawback of this latter approach is that this parallel interaction is not always 
optimum and that a certain amount of data must be extracted from the plant in real time in order to accomplish global learning.

The main focus of this paper is to prove that our adaptive fuzzy controller is capable of achieving a high accuracy and a good robustness through modifying the consequents of the rules in real time. The controller determines in which sense the rules have to be moved, that is, autolearning. The proposed methodology is robust against modification of the parameters of the plant (breakdowns). It is important to note that no initial knowledge about the control policy is required and therefore the fuzzy controller can start with a set of empty rules.

The controller proposed in this paper, besides presenting a very reliable control strategy easy to be implemented, has shown a remarkable improvement in saving energy. In the last decade, energy saving has been the subject of several scientific debates. Today, the energy saving has become a major human necessity that exceeds the economic interest; the climate change the planet suffers from and the need to reduce $\mathrm{CO}_{2}$ emissions resulting from energy production push research toward new and cleaner systems, hence, with less energy consumption. However, the qualities of organization and optimization that offer the intelligent control can contribute to the process of saving energy.

During the last ten years several studies have addressed the issue of energy saving using various techniques of intelligent control as adaptive algorithm, Genetic Algorithms, and Fuzzy Logic Controller [21-24] exploiting their qualities of optimization and organization that help to precise when and how to use the energy resources for ensuring the optimal use of energy. In [21] the energy consumption of air-condition of a building is done by an FLC that optimizes its parameters using a genetic algorithm, and the controller is in charge of modifying the set point based on the current variables detected in the controlled area at every moment; in fact, here the energy consumption is result of optimizing the set point value and not the air-condition feeding. The results achieved, using this technique, have been better than those obtained with the conventional automated control systems of buildings. In [22] a conventional FLC was used to manage multiunit air-conditioners to control the temperature of a classroom; thus, the energy consumption has been reduced 8.92\% compared to the traditional ON/OFF, and the FLC controller manages and organizes the operating mode of the fans. The rule base created by analyzing the effect of each unit of the multi-unit air-conditioners serves to clarify which are the appropriate units that have to operate avoiding the loss of energy produced off the use of the full system.

The adaptive fuzzy controller proposed in this paper has been able to reduce the energy consumption compared with the other used controllers in the same control process and under the same conditions. The difference between our technique and the techniques used in the bibliography is that our controller not only manages the time and the frequency of use of the actuator which consumes the energy, but also our adaptive fuzzy controller is capable to generate a very moderate output that feeds the actuator and ensures a reliable

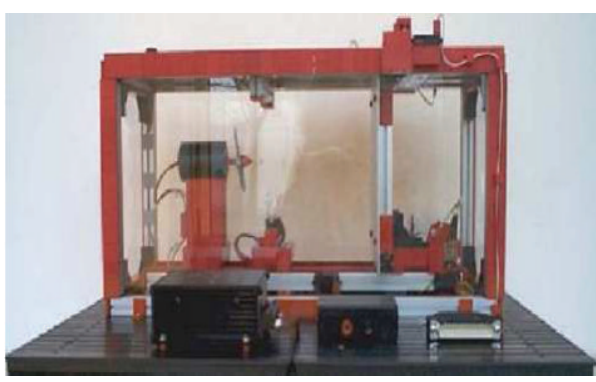

FIgURE 1: Scale model of a room.

TABLE 1: Formulas for the controller parameters in the ZieglerNichols closed-loop method.

\begin{tabular}{lccc}
\hline Structures & \multicolumn{3}{c}{ Parameters } \\
& $K_{P}$ & $T_{i}$ & $T_{d}$ \\
\hline$P$ controller & $0.5 K_{P_{u}}$ & $\infty$ & 0 \\
$P I$ controller & $0.45 K_{P_{u}}$ & $P_{u} / 1.2$ & 0 \\
$P I D$ controller & $0.6 K_{P_{u}}$ & $P_{u} / 2$ & $P_{u} / 8=T_{i} / 4$ \\
\hline
\end{tabular}

control with high smoothness and therefore consumes less energy.

This contribution has been organized as follows. The training board used in our experiment is briefly described in Section 2, followed by the design and the implementation of all the algorithms subject of this comparative study in Section 3. Section 4 presents the results obtained in real time for each algorithm, and also various comparisons between their performances will be presented. Conclusion and future works are drawn in Section 5.

\section{The Hardware Description}

In this paper we will try to simulate the control of temperature of a room using a training board with several intelligent control algorithms (Figure 1). Our goal is to maintain the temperature of the room at a desired value; the room is equipped with a temperature source that heats the environment and a fan for lowering the temperature in cases of over passing the set point. The controller will aim at generating the fan power signal and determining their performance rating with regard to the difference between the sensed temperature inside the room and the required temperature.

\section{Design and Implementation of the Used Controllers}

3.1. Tuning the PID Controllers. Before testing our fuzzy logic controller and the adaptive algorithm on the training board, we have tried to evaluate their behavior with the $P, I$, and $D$ controllers. Using the tuning method of Ziegler-Nichols in closed loop [25], we can easily tune our PID controller taking the values of parameters $P, I$, and $D$ from the predefined formulas Table 1 .

The Ziegler-Nichols closed-loop tuning method is a heuristic method of tuning a PID controller. It is performed 


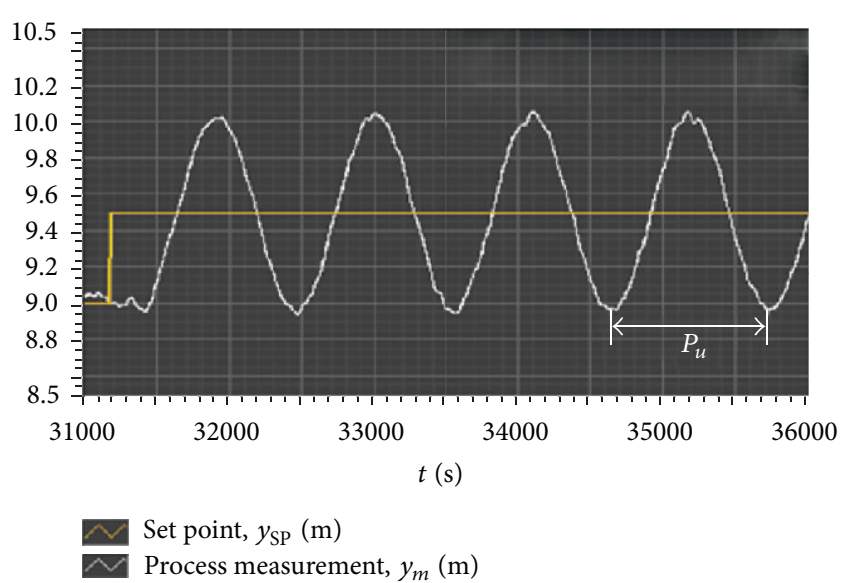

FIGURE 2: Ideal tuning phase of the Ziegler-Nichols closed-loop method.

by setting the $I$ (integral) and $D$ (derivative) gains to zero. The $P$ (proportional) gain is then increased (from zero) until it reaches the ultimate gain $K_{u}$, at which the output of the control loop oscillates with a constant amplitude. $K_{u}$ and the oscillation period $P_{u}$ are used to set the $P, I$, and $D$ gains depending on the type of controller used (Figure 2).

After setting the parameters of the PID controller we have processed to evaluate the performance of the $P I, P D$, and $P I D$ algorithms on our control device, and next we will see different responses of these algorithms.

3.2. Design of a Static Fuzzy Logic Controller. The TSK-0 fuzzy logic controller is a simple and stable controller, so it was used in this study (static controller), with two inputs and one output. The controller inputs are the temperature error and its derivative $\left(T_{e}, T_{\dot{e}}\right)$, and the error is the difference between the required temperature $T_{\mathrm{sp}}$ and the temperature at instant $i\left(T_{i}\right)$; in fact the error equation is $T_{e}=T_{\mathrm{sp}}-T_{i}$, and the error variations are calculated by the following expression: $\Delta T_{\dot{e}}=T_{\dot{e}_{k}}-T_{\dot{e}_{k-1}}$. The controller output is the tension that controls the fan operation. For a typical fuzzy control design, the membership functions of each variable divide the input space to present the different states that can take this input during the control process, and each one of these MFs have a linguistic label that describes the state of the input at this stage of control. In this paper, the membership functions are a set of triangular membership functions that describe the state of these inputs over the permitted range (five for the first input and seven for the second one). For the output, we are using a TSK-0 type, so it should be a set of scalar values that will be used as a fan alimentation. To do that, five scalar values are used as the output MFs $(0 \mathrm{~V}, 3 \mathrm{~V}, 5 \mathrm{~V}, 9.5 \mathrm{~V}, 15 \mathrm{~V})$, and every one of these values has a linguistic label that describes the operation state of the fan. Figure 3 presents the distribution of inputs and output of the proposed controller, and Table 2 presents the rules base of this controller; the rules contain the following expert knowledge (take rule 1 (Table 2) as an example):
IF actual temperature $\left(T_{e}=T_{\mathrm{sp}}-T_{i} \ll 0\right)$ is Extremely High

AND variation of error $\left(\Delta T_{\dot{e}}=T_{\dot{e}_{k}}-T_{\dot{e}_{k-1}}\right)$ is Decreasing Fast

THEN the fan speed has to be Medium $(u$, the Fan alimentation).

The entire used rules follow the standard form: IF $T_{e}$ is $S 1$ AND $\Delta T_{\dot{e}}$ is $S 2$ THEN $u$ is $U$.

The operation mechanism is given as follows. First, in order to get the degree of belief for each rule, one may let $T_{e}=$ $S 1$ and $\Delta T_{\dot{e}}=S 2$, and then $\mu_{\text {prex }}=\mu_{S 1} \Lambda \mu_{S 2}=\min \left(\mu_{S 1}, \mu_{S 2}\right)$, so the membership value of the IF part is given by $\mu_{\text {prex }}$, namely; $\mu_{u}=\mu_{\text {prex }}$. Then, the defuzzification method, the method of centre of gravity (3), is implemented to execute the defuzzification process to get the integration of all control signals.

After a deep study of the logical behavior of our plant, the adequate rules base was created to achieve the required set point.

3.3. Implementation of an Advanced (Adaptive SelfOrganizing) Fuzzy Logic Controller in Real Time. Generally, the plant to be controlled could be expressed mathematically by

$$
s(n+d)=f(s(n), \ldots, s(n-p), u(n), \ldots, u(n-q)),
$$

where $s(n)$ is the output of the plant at instant $n, d$ is the delay of the plant, $f$ is an unknown continuous and differentiable function, and $u$ is the controller output. In most engineering systems such as electrical networks, turbojet engines, modeling of signals, and nuclear reactors, a variable that plays a key role in establishing the optimum performance of the system can be found. This variable is the time delay. Hence, numerous researchers over the past years have shown a notable interest toward the problem of stability analysis and robust control for dynamic time-delay systems [26-28].

Generally, the controllability of the plant is a primary restriction, that is, that there always exists a control policy capable of translating the output to the desired value (within the operation range). This implies that the partial derivative of the plant output with respect to the control input is always different to zero. Therefore, as the plant is supposed to be continuous, it must have a constant monotonicity; that is, the relation between the plant output and the controller output must have a constant sign.

A set of IF-THEN fuzzy rules $R_{i_{1} i_{2} \ldots i_{N}}^{*}$ are used in the fuzzy control system having the following form:

$$
\begin{aligned}
& \text { IF } x_{1} \text { is } X_{1}^{i_{1}} \text { AND } x_{2} \text { is } X_{2}^{i_{2}} \\
& \text { AND } x_{N} \text { is } X_{N 1}^{i_{N}} \text { THEN } y=R_{i_{1} i_{2} \cdots i_{N}} \text {, }
\end{aligned}
$$

where $X_{V}^{i_{v}} \in\left\{X_{V}^{1}, X_{V}^{2}, \ldots, X_{V}^{n_{v}}\right\}$ with $n_{v}$ being the number of membership functions of the input variable $X_{V}$. For the output variable, $R_{i_{1} i_{2} \cdots i_{N}}$ is the numeric consequent of the rule (since singleton fuzzy rules are adopted for this work). In this paper, we used the product as T-norm for the fuzzy 


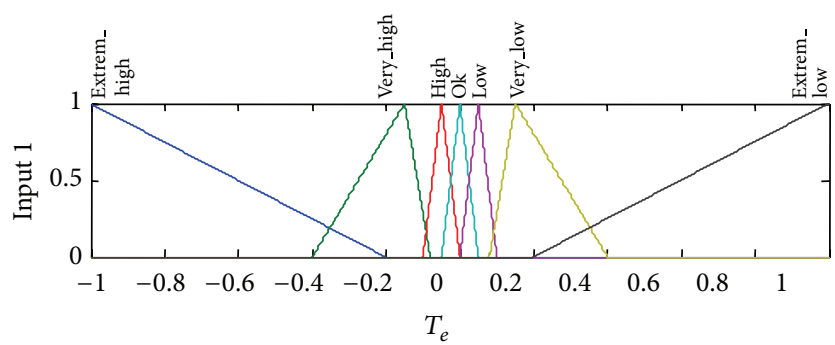

(a)

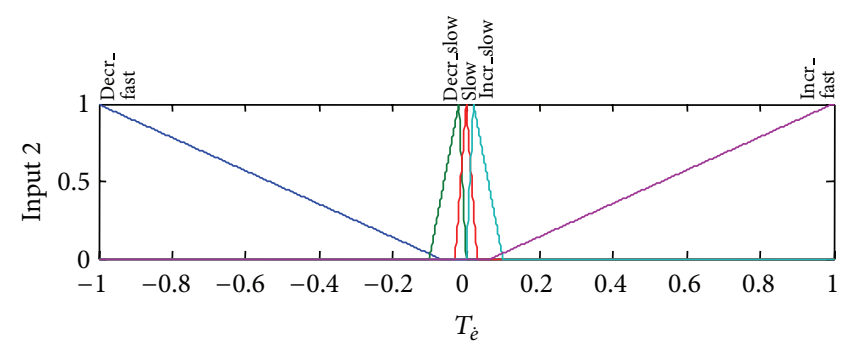

(b)

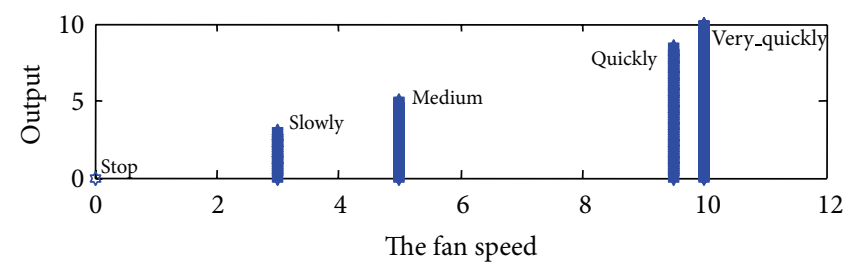

(c)

FIGURE 3: Distribution of inputs and outputs for a classical FLC.

TABLE 2: The Fuzzy Rules of the designed FLC.

\begin{tabular}{|c|c|c|c|c|c|}
\hline \multirow{2}{*}{$T_{e}$} & \multicolumn{5}{|c|}{$\Delta T_{\dot{e}}$} \\
\hline & Decr_Fast & Decr_Slow & Slow & Incr_Slow & Incr_Fast \\
\hline Extrm_High & Medium & Slowly & Slowly & Stop & Stop \\
\hline Very_High & Medium & Slowly & Slowly & Stop & Stop \\
\hline High & Medium & Slowly & Slowly & Stop & Stop \\
\hline Ok & Very_Quickly & Quickly & Medium & Slowly & Slowly \\
\hline Low & Very_Quickly & Very_Quickly & Quickly & Quickly & Quickly \\
\hline Very_Low & Very_Quickly & Very_Quickly & Very_Quickly & Quickly & Medium \\
\hline Extrm_Low & Very_Quickly & Very_Quickly & Very_Quickly & Quickly & Quickly \\
\hline
\end{tabular}

inference method and the "centre of gravity" with sumproduct operator as the defuzzification strategy. Using the previous notation, we can express the output of the fuzzy controller as follows:

$$
\widehat{F}\left(\vec{x}^{k}\right)=\frac{\sum_{i_{1}=1}^{n_{1}} \sum_{i_{2}=1}^{n_{2}} \cdots \sum_{i_{N}=1}^{n_{N}}\left(R_{i_{1} i_{2} \cdots i_{N}} \cdot \prod_{v=1}^{N} u_{x_{v}^{i_{v}}}\left(x_{v}^{k}\right)\right)}{\sum_{i_{1}=1}^{n_{1}} \sum_{i_{2}=1}^{n_{2}} \cdots \sum_{i_{N}=1}^{n_{N}}\left(\prod_{v=1}^{N} u_{x_{v}^{i_{v}}}\left(x_{v}^{k}\right)\right)},
$$

where $\vec{x}^{k}$ is the $N$-dimensional input vector at instant $k$.

3.3.1. Adaptation of the Rules Consequents. Recently, numerous works that try to improve the performance of control processes are based on adaptive controllers. Indeed, the intensive use of adaptive fuzzy controllers by the engineers in several applications is justified by the exceptional performance offered by these latter; hence, the adaptive controllers are capable of performing a self-adjustment of its parameters/settings without human intervention, which favors significantly the control processes improvement. In the literature we can find numerous adaptive mechanisms that serve the same purpose (enhance the performance of conventional controllers or static). In [29], an enhanced selforganizing fuzzy logic controller is proposed to control an active suspension system. The main feature of the original SOFLC used was the modification of the current fuzzy rules, and to suggest the suitable correction for the control input, the enhancement process is done by using a slidingmode control that provides a sliding input surface for the controller, while in [30], the sliding-mode control was used to perform an AFLC but this time without modifying the fuzzy rules and the secondary-controlled hydrostatic transmission system (SC-HST) can track the desired velocity with great effectiveness. In [31], the power network performance is controlled by using adaptive fuzzy logic controller driven by genetic algorithms that can significantly improve the control performance. The use of the GA and adaptive fuzzy sets reduces the system complexity and the number of rules. The proposed techniques have been able to assist the system operator in making control decisions to improve the voltage profiles in the system. Another example of adaptive and SOFLC is presented in [32], but this time the auto-learning process is done using a neural network that can modify the existent fuzzy rules or adding new ones. The control of a nonlinear chaotic system using this technique was very 


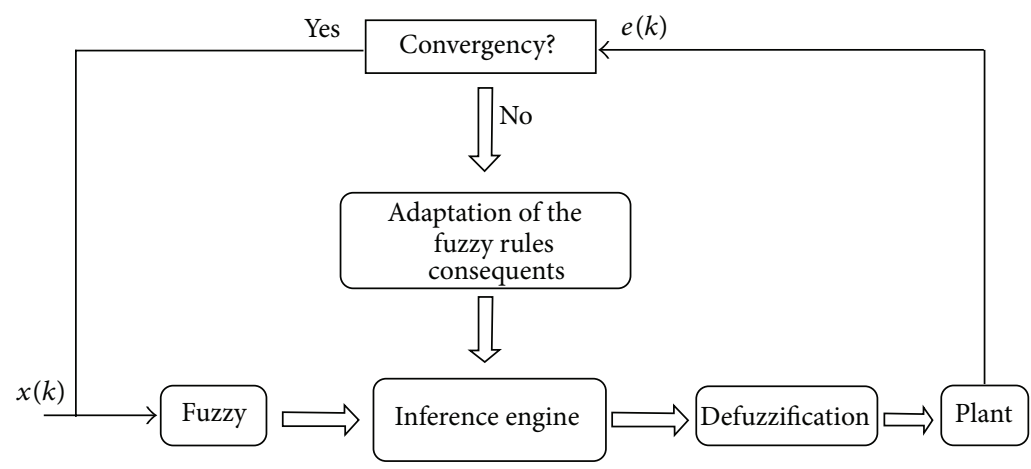

FIGURE 4: Basic structure of an adaptive fuzzy controller.

successful; hence, the new fuzzy rules used by the FLC driven by the neural network are able to optimize the output of the controller.

In our approach, we try to present an adaptive algorithm that will be able to adapt the controller parameters using the information obtained from the current error in the output of the plant. The correction sense is deduced from the monotony of the plant. Using the same structure of the static fuzzy logic controller mentioned earliar but with no definite rules, we will try to implement our adaptive control system. The main problem that real-time control strategies must face lies in the fact that, as the internal functioning of the system to be controlled is unknown, we are unaware of how to modify the controller's parameters.

In order to get more accuracy in the dynamics of the system it is essential to know which rules are responsible leading the plant to be stable in the desired state. Learning occurs when the algorithm starts handling the parameters (in this case the conclusions of the rules of the main controller) in an attempt to ameliorate its control behavior. In this case, the system learns in such a way that the acquired knowledge is reflected in the new values of the rules. Figure 4 resumes the basic structure of the proposed adaptive fuzzy logic controller.

In order to build an accurate self-organizing controller, the use of the information on how the plant output varies with respect to the control signal for every possible operating region is essential. This involves knowing the Jacobian matrix of the function of the plant. Unfortunately, this information is usually not available in a control task. Consequently, it is clear that because of the type of information available from the plant (its monotonicity sign and its delay) only a relatively coarse control can be applied to the system. In the algorithm subject of this study, coarse adaptation of the fuzzy rule consequents is achieved by evaluating the current state of the plant and proposing a correction of the rules responsible for the existence of such a state, either as a reward or as a penalty, in the following way:

$$
\begin{aligned}
\Delta R_{i_{1} i_{2} \cdots i_{N}}(k) & =C \cdot \mu_{i_{1} i_{2} \cdots i_{N}}(k-d) \cdot e_{y}(k) \\
& =C \cdot \mu_{i_{1} i_{2} \cdots i_{N}}(k-d) \cdot(r(k-d)-y(k)),
\end{aligned}
$$

where $\mu_{i_{1} i_{2} \cdots i_{N}}$ is the strength or $\alpha$-level of rule $R_{i_{1} i_{2} \cdots i_{N}} \cdot e_{y}(k)$ is the error at instant $k$.

Since, as in [33], the degree of activation of the rule that leads to achieve the control output $u(k-d)$ was used proportionally with the modification adopted at instant $k$. In the aforementioned expression, $d$ represents the time delay, $r(k-d)$ is the required set point of the plant output at instant $k-d$, and $y(k)$ is the current plant output. For best understanding and to ensure that this modification is always in the direction of a better positioning of the rule consequents, let us suppose that the plant output increases with control input. So if the required set point at instant $k$ is bigger than the output, then $u(k-d)$ would have to be made bigger. Consequently, $C$ will have a positive sign and the correction to the rules responsible for having obtained $u(k-d)$ should be changed proportionally to the current plant error. At this point, it is very important to clear up that using $r(k)$ would be incorrect, because the rules that are activated at instant $k-d$ serve to achieve the desired value $r(k-d)$ and not $r(k)$.

The determination of the absolute value of the coefficient $C$ is calculated spent in offline using the formula: $|C|=$ $\Delta u / \Delta y$, where $\Delta y$ is the operation range of the plant output, which must be estimated beforehand from the knowledge about the set points that we are going to use, and $\Delta u$ is the operation range of the controller's actuator. In our case and after studying our plant we chose $|C|=15$.

3.3.2. Adaptation of the Rules Antecedents. The methodology cited earliar allows us to tune only the consequents of the fuzzy rules. The emplacement and the form of the membership functions has a big influence on the control performance, so to achieve a high level of accuracy during a control process it is very important to optimize them. To solve this problem we intend to find a new configuration of MF which ensures a homogeneous distribution of a certain performance criterion across the operating regions. The performance criterion which would be used in this optimization is the integral of the square error (ISE). Thus, the region that has a big contribution to the ISE is the region more frequently activated. The equation for the contribution to the ISE of the 
operation regions between centers $j$ and $j+1$, of input variable $X_{i}$, is

$$
\begin{aligned}
\operatorname{ISE}_{i}^{j}=\frac{1}{\sigma_{y}^{2}}( & \left.\int e^{2}(t) d t\right|_{x_{i}(t) \in\left[c_{i}^{j-1}, c_{i}^{j}\right]} \\
& \left.-\left.\int e^{2}(t) d t\right|_{x_{i}(t) \in\left[c_{i}^{j}, c_{i}^{j+1}\right]}\right),
\end{aligned}
$$

where $x_{i}$ is the $i$ th component of the input vector, and $C_{i}^{j}$ is the position of the centre of the $j$ th membership function of the $i$ th input variable. The first integral calculates the contribution to the ISE when the input is within the range $[(j-1), j]$. The second integral calculates that contribution when the input is within the range $[j,(j+1)] . \sigma_{y}^{2}$ is the variance of the plant output and is used as a normalization factor. In the discrete domain, real cases, this equation can be simplified replacing the integral sign by the summation sign.

From (5) when the error in the left region was bigger than in the right, the contribution to the ISE would be positive; thus to compensate this, the centre has to be moved to the left. Oppositely, if it is negative the centre has to be moved to the right. The movement of the centers of membership functions should not change the original order of the membership functions during all the process. The following equation describes how the movement of the centre can be calculated to preserve the order of the centers and locate them in the suitable places where their contribution to the total ISE can be homogeneously distributed (the $i$ index has been removed for the sake of simplicity):

$$
\Delta c^{j}= \begin{cases}\frac{c^{j-1}-c^{j}}{2} \frac{\mathrm{ISE}^{j}}{\operatorname{ISE}^{j}+1 / R^{j}}, & \text { if } \operatorname{ISE}^{j} \geq 0, \\ \frac{c^{j+1}-c^{j}}{2} \frac{\left|\mathrm{ISE}^{j}\right|}{\left|\mathrm{ISE}^{j}\right|+1 / R^{j}}, & \text { if } \operatorname{ISE}^{j},\end{cases}
$$

where $R$ is the responsible parameter that precise; how far the centre can be moved without surpassing the allowable ranges of each used membership functions. For high values of $R$, the centre can make a very large displacement, while for low $R$ the movement is very limited. In the learning process that we will use in this work, we start with high values for $R$ (100) for all centers and decrease them every evaluation of the ISE dividing by two. Every $N_{\text {ISE }}$ iterations the sum should be calculated. Firstly, the consequents are tuned during one learning run without calculating the ISE. In the next run the ISE would be calculated, and the placement of the membership function is changed at the end of the run using (6). In the next run and without the ISE the output consequents are tuned. After that the ISE is calculated periodically. Thus $N_{\text {ISE }}$ has the size of one learning run and the ISE is calculated every $N_{\text {ISE }}$ iterations. After $N_{\text {ISE }}$ iterations of the control process and this time with a smaller $R$ the ISE is computed again.

\section{Simulation Results}

4.1. Real Simulation: Temperature Control. In real simulation, we will use a training board to control the temperature using the algorithms we have formerly designed. All the experiments were carried out under the same conditions. Due to the nature of the results obtained from the controlled process, the comparison based on graphical representations is slightly blurred. In fact we proceed to a short quantitative analysis based on mean squared error (MSE). The MSE calculated here is not the MSE of the function approximation by the controller but it is the MSE between the set point and the plant output measured after $d$ instants of time, being $d$ the delay of the plant. Consider

$$
\text { MSE }=\frac{\sum_{K=1}^{\text {Num_Epochs }}(r(k)-y(k+d))^{2}}{\text { Num_Epochs }} .
$$

4.1.1. Temperature Control Using a P, I, and D Control Policy. The tuning mechanism for the controllers is based on PID structure. Through Ziegler-Nichols closed-loop method and using their formulas, we can establish the values of $P, I$, and $D$ for different structures. Subsequently, the results for each structure are shown.

The PD Controller. The PD controller has been able to control the temperature, the MSE (mean squared error) in this case is around 0.63 for the first 40 minute, and Figure 5 shows the control evolution in this case for various set points.

The PI Controller. The PI controller shows the same behavior as the $P D$, and it is also able to control the temperature with some kind of improvement. MSE (mean squared error) in the last 40 minute is around 0.43 . Figure 6 presents the $P I$ response for various set points.

Using a Full PID Controller. It should be noted here that in our experiment we are controlling the fan that would decrease the temperature. Temperature increasing in case of change of set point at a temperature higher than the current is done by stopping the fan, allowing the system heats only (heating process), so that achieving the new higher set point needs a long relative time and affects the accuracy at this phase due to the poor performance of the bulb (temperature source).

Figure 7 clearly shows that our PID works well as a controller for various values of set points, guaranteeing the convergence around the set point with a $0.47 \mathrm{MSE}$ in the last 40 minutes. The results obtained from the PID and PI controllers are almost identical. In fact, in this case a $P I$ controller can be used instead of a full PID. Formerly it has been shown that the controllers based on $P, I$, and $D$ can also control the temperature, although they obtained different MSE and they show some limitations (relative large error and time control). Next section will show the performance of a conventional static fuzzy logic controller on this plant.

4.1.2. Temperature Control Using a Static Fuzzy Logic Controller. Figure 8 presents the general behavior of the fuzzy logic controller during 200 minutes for various set points, showing that this controller is able to decrease temperature 


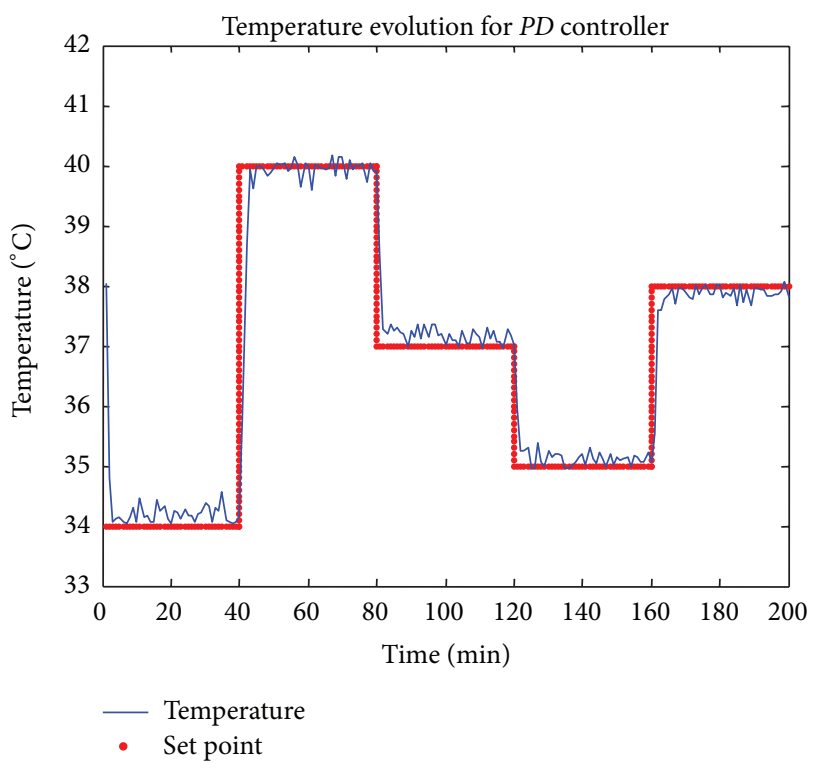

FIgURE 5: Control evolution with $P D$ algorithm for various set points.

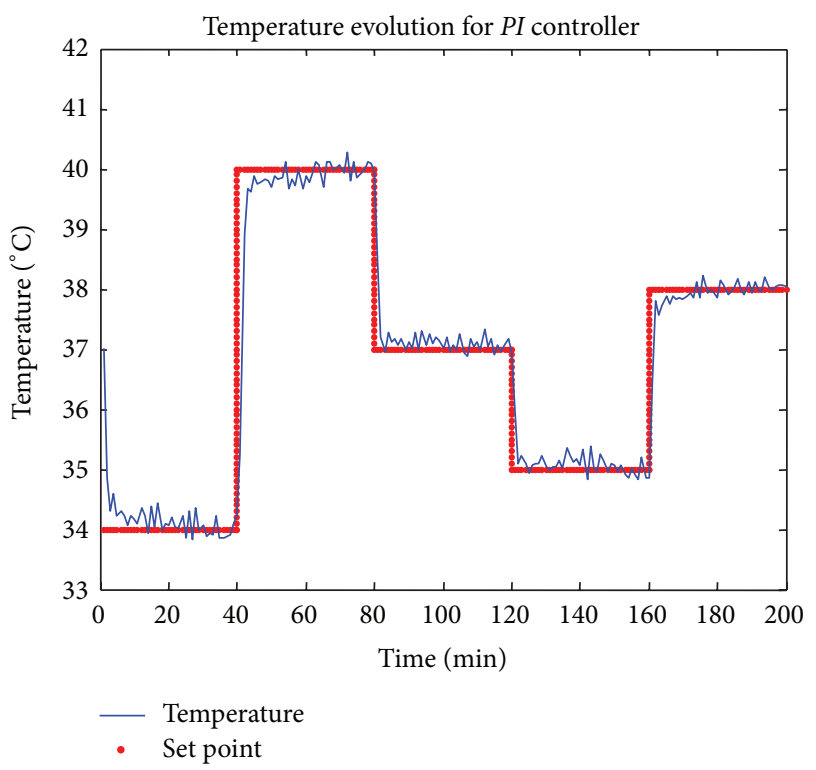

FIgURE 6: Control evolution with PI algorithm for various set points.

from $41^{\circ} \mathrm{C}$ to around $34^{\circ} \mathrm{C}$, presenting a reasonable error committed during this control process. A good control time control and a smaller error than the PID algorithms reflect the good performance of this kind of controllers. The MSE for the last 40 minutes is 0.31 .

4.1.3. Temperature Control Using Adaptive Fuzzy Logic Controller (Rules Adaptation). The proposed modification on the operating mode of the fuzzy logic controller has to be tested to make out its effect on the controller performance; this modification is a process of adaptation and self-learning. The rules will be able to adapt and learn how to react in order to

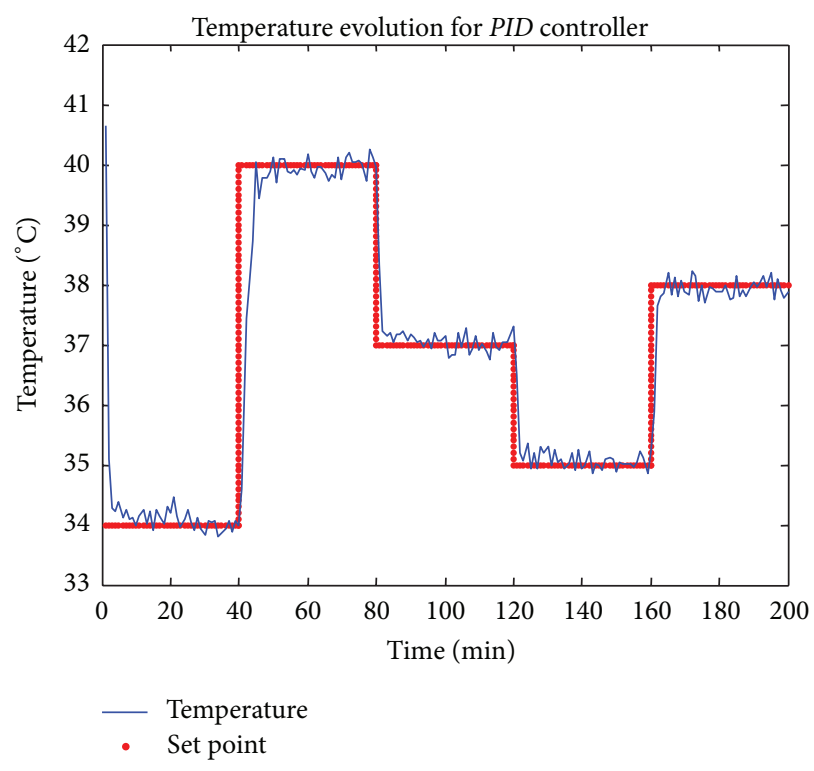

FIgURE 7: Control evolution with PID algorithm for various set points.

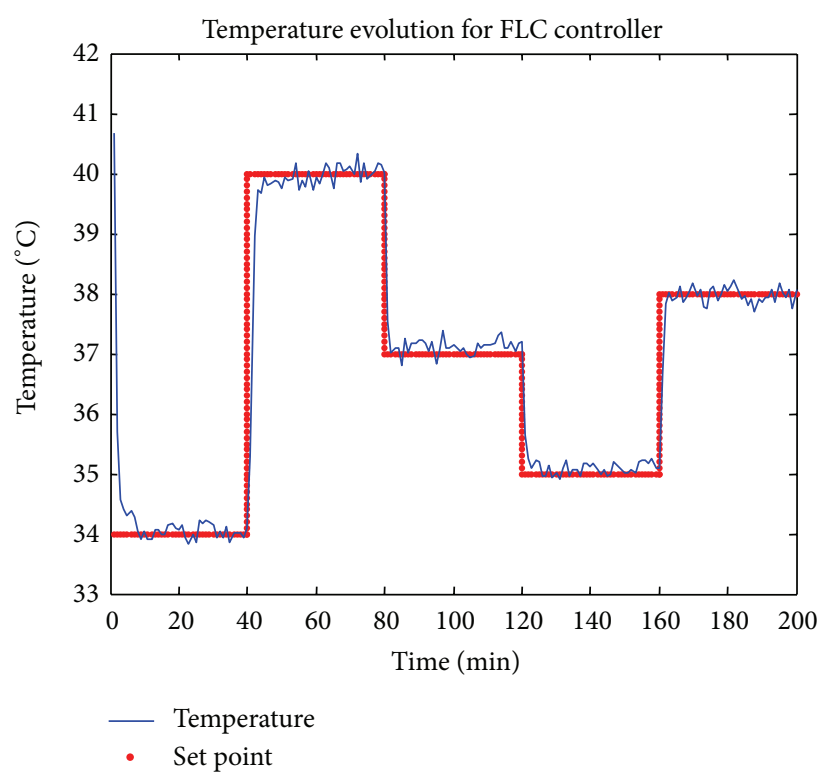

Figure 8: Control evolution with FLC algorithm for various set points.

achieve a minimum error starting from empty rules or with random values using the information from the previous state of error [33]. In other words, it gets the sense of variation of the controller and applying the adequate correction to the rules in a reward/penalty manner. Figure 9 shows the attitude of the proposed algorithm to control the temperature for different values of set points.

Like all the control algorithms that we tested earlier, our adaptive fuzzy logic controller has shown a good performance in controlling the temperature, following the different changes at the set points. An analysis of the MSE in the last 40 


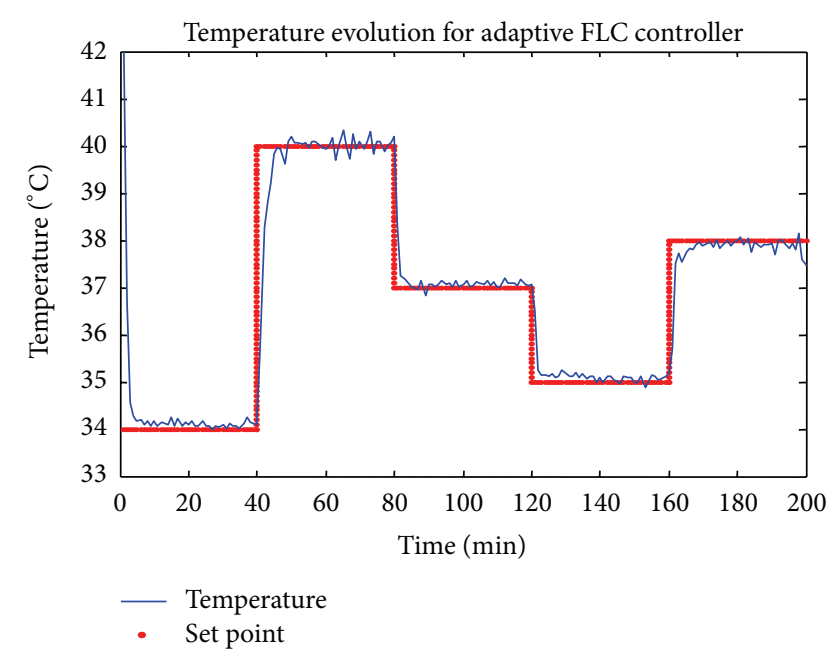

Figure 9: Control evolution with adaptive FLC for various set points.

minutes shows clearly the superiority of the latter compared with others; the adaptive algorithm has been able to reduce the error to almost $50 \%$. The MSE in this case is 0.24 .

4.2. Results Comparison. All the algorithms used in this contribution were able to carry out the control process, but what really makes the difference between them is the error during the regulation process. The graphic comparison of the results does not clarify the difference between the precision of each algorithm used, due to the nature of the response of the system. In fact, in order to show clearly the difference between each one of them, we used two comparison methods (graphical and numerical) based on the MSE obtained.

The graphical method consists in drawing the representing curve of the difference between the errors committed by two different algorithms at the same time interval and to achieve the same set point, that is represents the function defined as

$$
\begin{aligned}
& \text { error difference }=E_{(\text {Algorithm 1) }}-E_{(\text {Algorithm 2) }} \\
& \text { if } E_{(\text {Algorithm 1) }}>E_{(\text {Algorithm 2) }} \rightarrow \text { Error difference }> \\
& 0 .
\end{aligned}
$$

The graphical representation of the function error difference is above zero. Consider

$$
\begin{aligned}
& \text { if } E_{(\text {Algorithm 1) }}<E_{(\text {Algorithm 2) }} \rightarrow \text { Error difference }< \\
& 0 .
\end{aligned}
$$

The graphical representation of the function error difference is under zero.

In Figure 10 we can see the graphical representation of the difference between the error committed by the adaptive algorithm and the error committed by the other algorithms used during the first 40 minutes.

The four cases presented in Figure 10 show that the error difference curve is almost always under the zero line, and mathematically this means that
TABLE 3: Comparative performance indices for all used algorithms in real simulation.

\begin{tabular}{lccccc}
\hline \multirow{2}{*}{ MSE } & \multicolumn{4}{c}{ Algorithms } \\
& $P I$ & $P D$ & PID & Classic FLC & Adaptive FLC \\
\hline MSE $_{160 \text { to } 200}$ & 0.43 & 0.49 & 0.47 & 0.31 & 0.17 \\
MSE $_{100 \text { to 200 }}$ & 0.38 & 0.44 & 0.41 & 0.28 & 0.23 \\
MSE $_{\text {0 to 200 }}$ & 0.84 & 0.89 & 0.85 & 0.78 & 0.67 \\
\hline
\end{tabular}

TABle 4: Comparative performance indices before and after the adaptation of consequents.

\begin{tabular}{lcc}
\hline & MSE $_{\text {Total }}$ & MSE $_{100 \text { to } 200}$ \\
\hline Adaptation of consequents & 0.67 & 0.23 \\
Adaptation of consequents and antecedents & 0.53 & 0.17 \\
\hline
\end{tabular}

$$
\begin{aligned}
& \text { error difference }=E_{(\text {Algorithm 1) }}-E_{(\text {Algorithm 2) }}<0 \text {; } \\
& \text { implies that } E_{(\text {Algorithm 1) }}<E_{(\text {Algorithm 2) }} \\
& \text { in our case: } E_{(\text {Algorithm 1) }} \text { is } E_{\text {(Adaptive) }} \text {. }
\end{aligned}
$$

The error difference is almost always less than zero (error difference $<0$ ) in the four cases, which means that the $E_{\text {(Adaptive) }}$ is less than the rest of committed errors by others algorithms $E_{(F L C, P I D, P D, P I)}$.

The numerical comparison is based on the analysis of the MSE for each algorithm. Table 3 shows the MSE of each algorithm used in this study during the last 40 minutes. The MSE is calculated for all time steps of the simulation. The second index is also as MSE but now only calculated for the time from 100 to 200 minutes.

The results presented in this table show the differences between the algorithms used. The adaptive controller has the best error reduction compared to the other; that is, the adaptation presented and tested is a good alternative to replace the $P, I$, and $D$ controllers or a classical fuzzy logic controller for these kinds of plants.

4.3. Adaptation of the Consequents and the Antecedents of the Fuzzy Rules. Previously we have presented a new methodology for the adaptation of the fuzzy rules consequents in real time to improve the performance of the FLC, and the experimental results show the great contribution that brings this technique. Basically we know that the correction provided by an FLC is extracted essentially from the consequents and antecedents of the fuzzy rules. In fact, after the remarkable improvement obtained by modifying the rules consequents of our FLC, we have proceeded to optimize their antecedents in order to achieve the maximum stability and more accuracy. Knowing that the values of the rules antecedents are calculated directly from the membership functions of the inputs variables, however, the location of the latter will influence the determination of these values. The optimization of the fuzzy rules antecedents is done by the redistribution of the centers of MFs maintaining the equilibrium of the contribution of the ISE in all the input space as is previously explained. At next will see the results obtained after the adaptation of the consequents and antecedents of the fuzzy rules. 


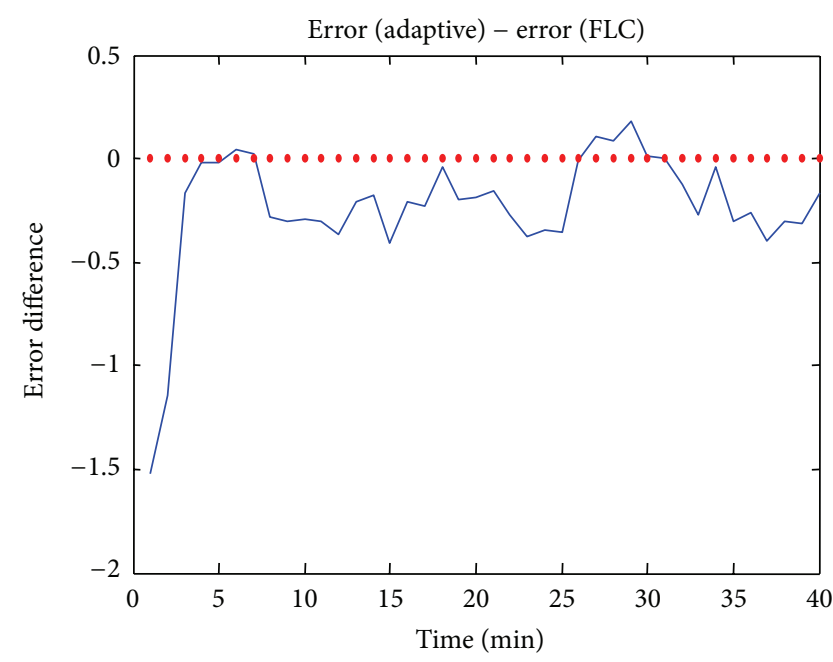

(a)

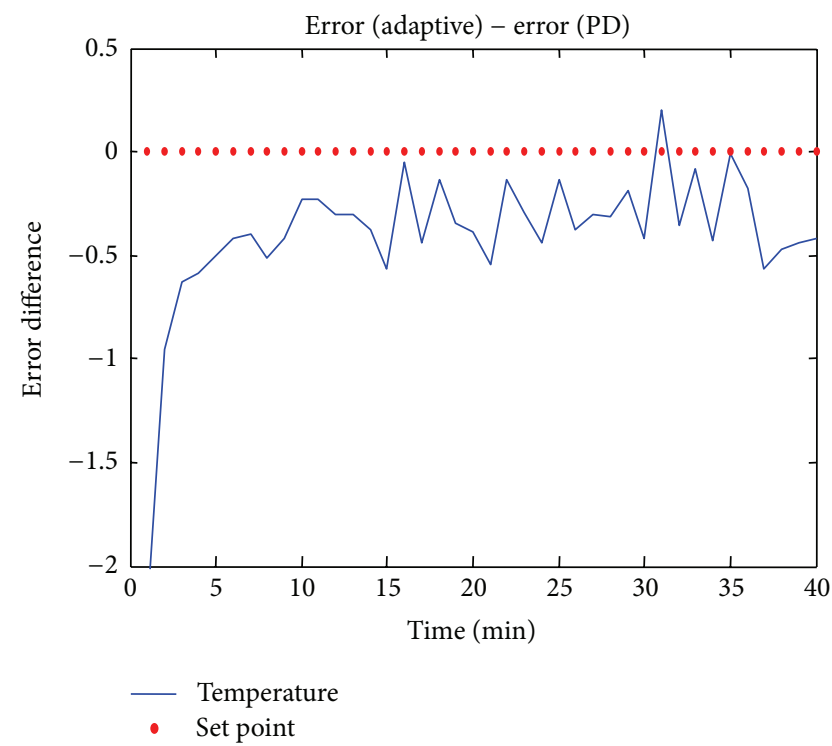

(c)

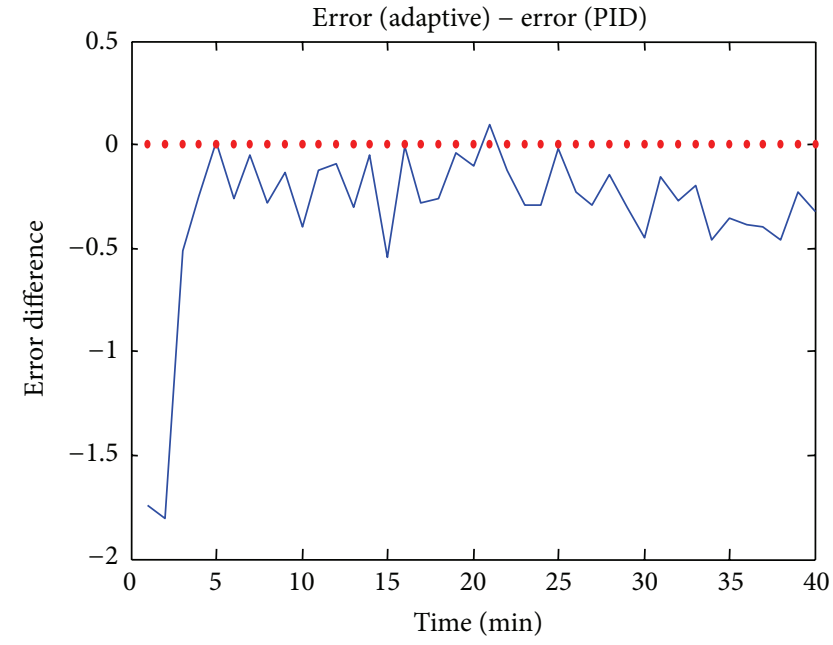

(b)

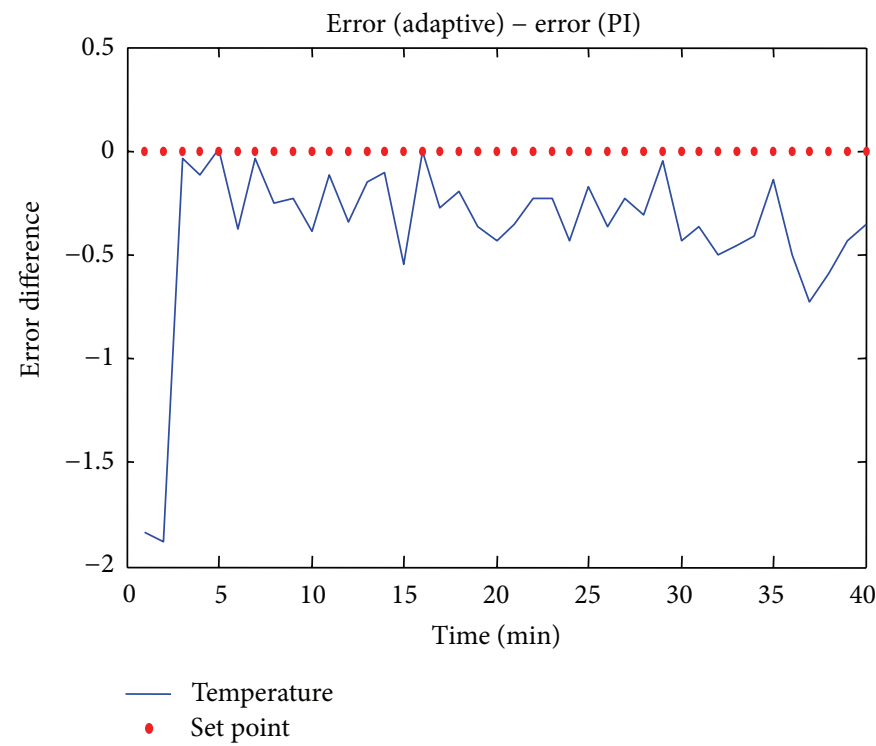

(d)

FIGURE 10: Differences between the error committed by the adaptive algorithm and the remaining algorithms.

TABLE 5: Comparative performance indices for all used algorithms.

\begin{tabular}{lcc}
\hline & MSE $_{\text {Total }}$ & MSE $_{100 \text { to 200 }}$ \\
\hline PD controller & 0.89 & 0.44 \\
PI controller & 0.84 & 0.38 \\
PID controller & 0.85 & 0.41 \\
FLC controller & 0.78 & 0.28 \\
Full adaptive FLC controller & 0.53 & 0.17 \\
\hline
\end{tabular}

Figures 11 and 12 represent, respectively, the evolution of temperature and the new location of the membership functions after introducing the optimization of the antecedents of the rules to the process of adaptation. We can observe that the evolution of temperature in this case is smoother and the
TABLE 6: Comparative performance indices before and after the perturbation.

\begin{tabular}{lcccc}
\hline $\begin{array}{l}\text { MSE in } 10 \\
\text { minutes }\end{array}$ & $\begin{array}{r}+25 \% \text { in the power of } \\
\text { the fan } \\
\text { Adaptive } \\
\text { FLC }\end{array}$ & FLC & $\begin{array}{c}-20 \% \text { in the power of } \\
\text { the fan } \\
\text { FLC }\end{array}$ \\
\hline $\begin{array}{l}\text { Before } \\
\text { perturbation } \\
\begin{array}{l}\text { After } \\
\text { perturbation }\end{array}\end{array}$ & 0.78 & 0.42 & 0.64 & 0.1 \\
\hline
\end{tabular}

error is relatively smaller than the case of adapting only the consequents of the rules.

This new policy of control shows great improvements again and this time with more smoothness. Table 4 presents 
TABLE 7: Comparative indices of the electrical consumption for each used algorithm.

\begin{tabular}{cccccc}
\hline & $P I$ & $P D$ & $P I D$ & FLC & Adaptive FLC \\
\hline Electrical cost & 1776.3 & 1599.3 & 1601.55 & 1621.3 & 1384.1 \\
\hline
\end{tabular}

TABLE 8: The real results of MSE and EC with various sampling time using adaptive FLC.

\begin{tabular}{lccccc}
\hline & $T_{s}$ & $3 T_{s} / 2$ & $2 T_{s}$ & $5 T_{s} / 2$ & $3 T_{s}$ \\
\hline MSE & 0,58 & 1,21 & 1,6 & 2,63 & 6,92 \\
EC & 644,33 & 460,6 & 343,36 & 282,7 & 276 \\
\hline
\end{tabular}

the MSE indices of adaptive controller in both cases (the adaptation only of consequents and adaptation of the consequents and antecedents) during all the control process and during the last 100 minutes.

Finally Table 5 summarizes the results of all MSE obtained during the simulation of all algorithms tested in this study, showing the differences in precision between all used algorithms.

4.4. The Perturbation Effects on the Behavior Algorithm. Figure 13 depicts the behavior of the algorithms (static FLC and adaptive FLC) after a disturbance of $25 \%$ at minute 20. The disturbance used here is a simulation of a temperature decrease caused by a secondary fan, and at the 65 minute, we have caused a disturbance of $20 \%$ but this time we simulate a temperature increase caused by another source of temperature. The response of the plant after the disturbances for each algorithm can clearly explain the differences between every one of them. Figure 13(a) shows the behavior of static FLC in perturbation cases. We can notice how these disturbances can affect the control precision; the static FLC keeps the plant under control but with large error without overpassing the perturbation effects.

In the second graph, the adaptive FLC presents a very good performance against these disturbances. It can be clearly noticed that the control precision does not suffer big changes and that the adaptive algorithm can overcome the perturbation effects in few moments later; it can correct the new error and return it within the allowable range. Thus, Table 6 presents a numerical comparison between the error committed in 10 minutes before causing the disturbances and the error committed in 10 minutes with disturbance for each algorithm. This comparison is correct while the 10 minutes used in the calculation of the MSE before and after the perturbation are always from the same set temperature.

It is important to make clear here that the error reduction in the first case of perturbation does not mean a control improvement, but it is due to the new fan added by simulation ( $+\% 25$ in the power of the fan), and adding a fan with power more bigger signifies big reduction in the error committed and may be the error will change the sign if this fan power was too much.

The MSE presented in this table proves that the perturbation effects on the behavior of the adaptive algorithm are very small when compared with their effects on the behavior of

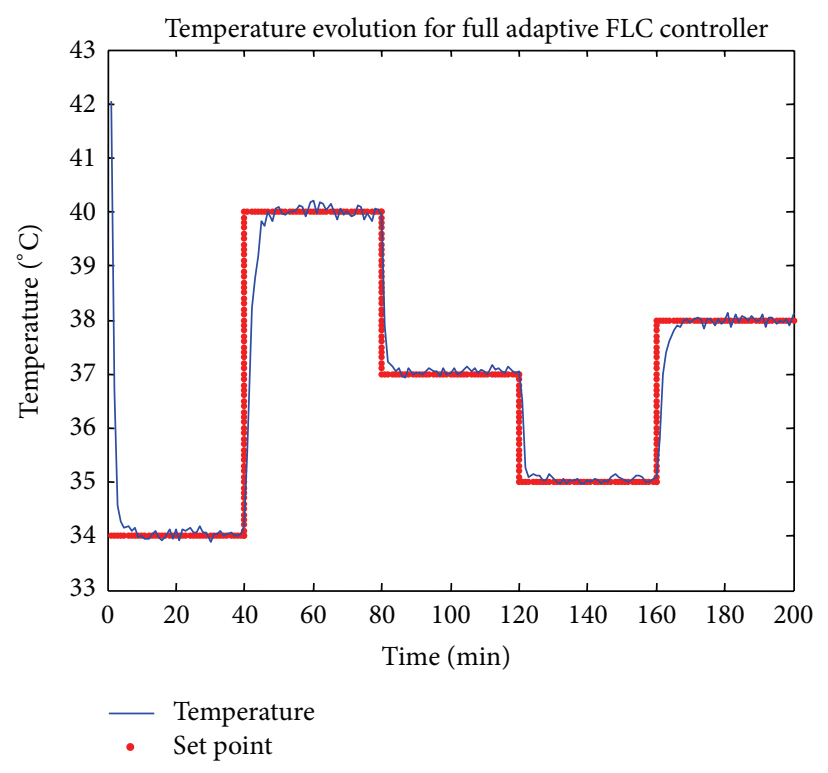

FIgURE 11: Control evolution with Full adaptive FLC for various set points.

the FLC algorithm. The adaptive FLC can quickly compensate the new environmental variations by maintaining the error within the allowable range, whereas the FLC cannot adjust to the new environmental variations. Therefore, the control precision suffers from serious changes.

4.5. An Overview on the Electric Consumption of Each Algorithm. An intelligent control system that provides high accuracy and strong stability against several types of disturbances is considered as a good control policy. Very large applications that require control policies are used in industrial applications where the best performance is not the only criterion of election, and the cost of the used techniques also plays important role in this election. In fact, the best control policy would be able to ensure a good combination between the performance advantage and a moderate cost. In this temperature control process the full adaptive FLC has proved it's superiority showing the best control performance.

It is high time to ask now, what cost will this technique need?

For this reason we will see the electric consumption of each control algorithm used in this paper. The simplest way to calculate the power consumed by active or resistive load connected to a single phase circuit (DC or AC) is multiplying the value of the applied voltage in volts $(V)$ by the value of the intensity $(I)$ of the current that runs through it in amperes (A). This calculation is performed using the following formula:

$$
P=(V \times I) .
$$

Figure 14 shows the electric consumption of all used algorithms during 200 minutes through various set points. In the minutes 0 to 40,80 to 120 , and 120 to 160 , the control system would have to drop the temperature to achieve the set 
TABLE 9: The MSE and the electrical consumption (EC) obtained during each simulation.

\begin{tabular}{|c|c|c|c|c|c|c|c|c|c|c|c|c|c|}
\hline & & \multicolumn{6}{|c|}{ Set points 1} & \multicolumn{6}{|c|}{ Set points 2} \\
\hline & & 1 & 2 & 3 & 4 & 5 & $\sum / 5$ & 1 & 2 & 3 & 4 & 5 & $\sum / 5$ \\
\hline \multirow{2}{*}{$P I$} & MSE & 1.62 & 1.09 & 1.56 & 1.36 & 1.7 & 1.46 & 1.22 & 1.28 & 1.35 & 1.7 & 1.65 & 1.45 \\
\hline & $\mathrm{EC}$ & 1142 & 968.4 & 1159.9 & 1136.7 & 1158 & 1113 & 1112 & 1108 & 1106 & 1092 & 1121.3 & 1107.8 \\
\hline \multirow{2}{*}{$P D$} & MSE & 1.07 & 1.07 & 1.5 & 1.12 & 1.45 & 1.25 & 1.37 & 1.28 & 1.3 & 1.35 & 1.4 & 1.35 \\
\hline & $\mathrm{EC}$ & 1048.2 & 1022 & 1163.4 & 1024.3 & 1109.3 & 1074 & 1092 & 1120.83 & 1133 & 1089 & 1079.9 & 1103 \\
\hline \multirow{2}{*}{$P I D$} & MSE & 1.07 & 1.16 & 1.66 & 1.35 & 1.06 & 1.26 & 1.34 & 1.27 & 1.26 & 1.32 & 1.26 & 1.3 \\
\hline & $\mathrm{EC}$ & 1121.1 & 1120.2 & 1151.9 & 1135.2 & 1133.1 & 1132.3 & 1127.3 & 1102.04 & 1101.1 & 1179.3 & 1121.7 & 1126.3 \\
\hline \multirow{2}{*}{ FLC } & MSE & 0.92 & 1.03 & 1.49 & 1.31 & 1.05 & 1.12 & 1.47 & 1.08 & 1.11 & 1.33 & 1.3 & 1.25 \\
\hline & $\mathrm{EC}$ & 1159.3 & 1140.3 & 1130.9 & 1139.5 & 1108.7 & 1135.7 & 1121 & 1098.2 & 1107.4 & 1115.2 & 1162.3 & 1120.8 \\
\hline \multirow{2}{*}{ Adapt FLC } & MSE & 1.1 & 0.7 & 0.74 & 0.65 & 0.5 & 0.74 & 1.1 & 1 & 0.83 & 0.78 & 0.84 & 0.9 \\
\hline & $\mathrm{EC}$ & 1084.4 & 1088.2 & 1084.3 & 932 & 1082.3 & 1054.2 & 1187 & 1121.3 & 1009.7 & 1013.8 & 987.5 & 1063.8 \\
\hline
\end{tabular}

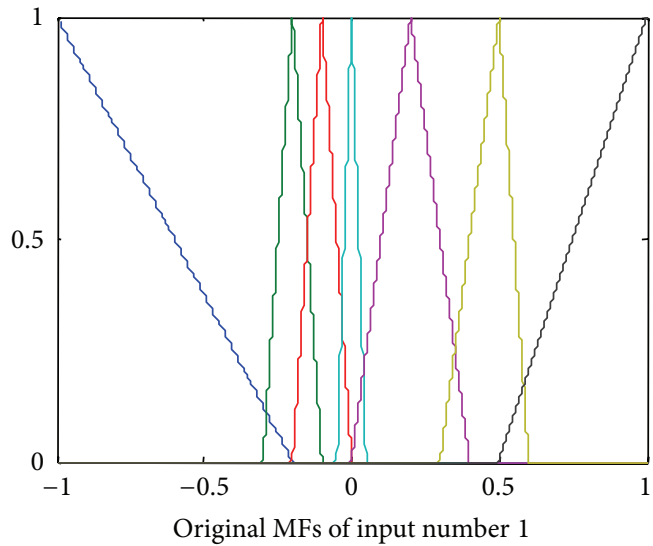

(a)

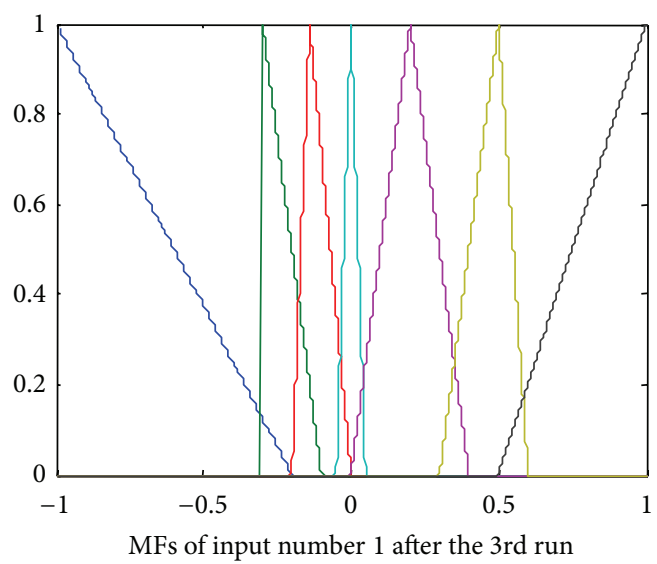

(c)

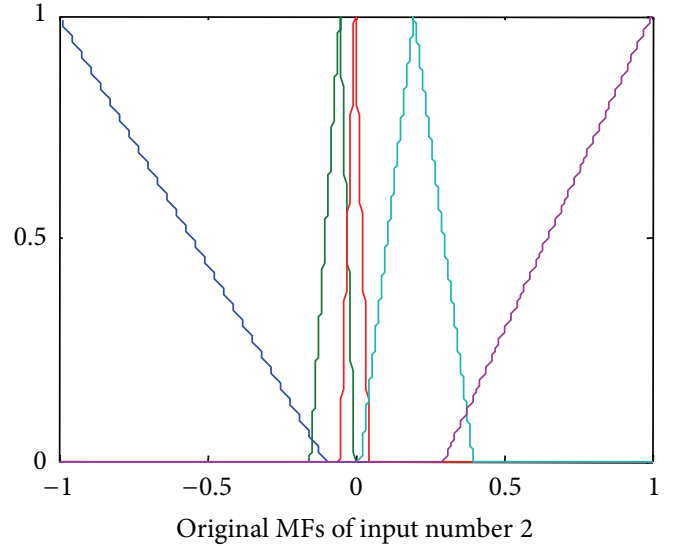

(b)

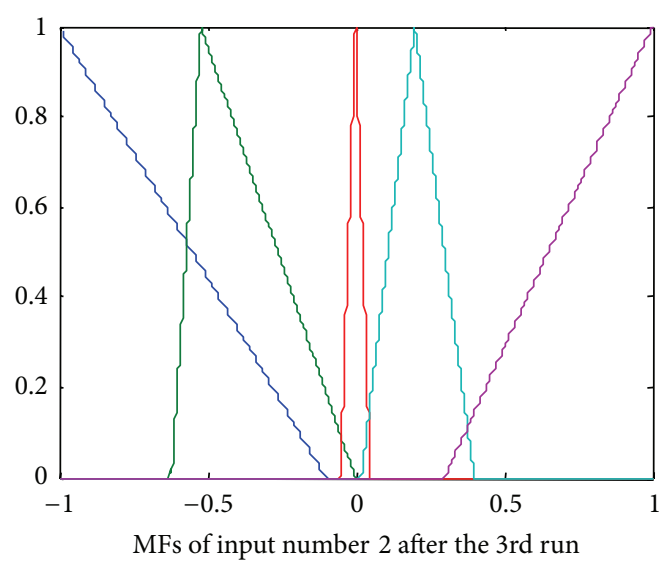

(d)

FIGURE 12: The inputs membership function before and after full adaptation.

point; in fact, the fan must operate continuously to set the temperature. At the intervals 40 to 80 and 160 to 200 , the set point is greater than the current temperature which means that the fan has to be turned off to warm the atmosphere and re-operate when the temperature overpasses the required set point. We recall here that the controller output is the fan feed.
Analyzing the results presented in Figure 14 we can easily deduce that the signal generated by the adaptive algorithm is more moderate than other algorithms. For the rest of algorithms, the output signal oftentimes touches the extremes ranges of the actuator which mean a high consumption of current, thus forcing the fan to operate brutally; it has to 


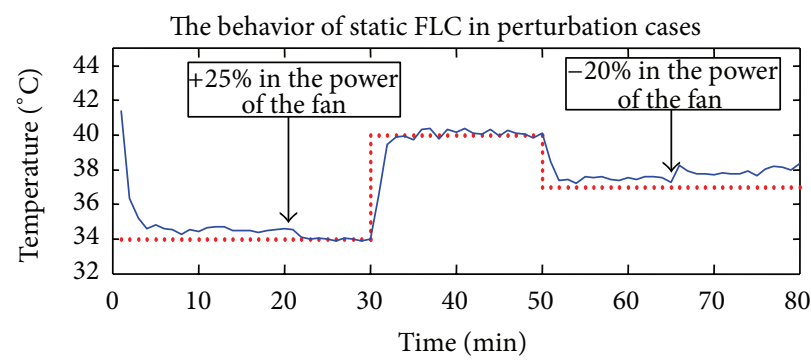

(a)

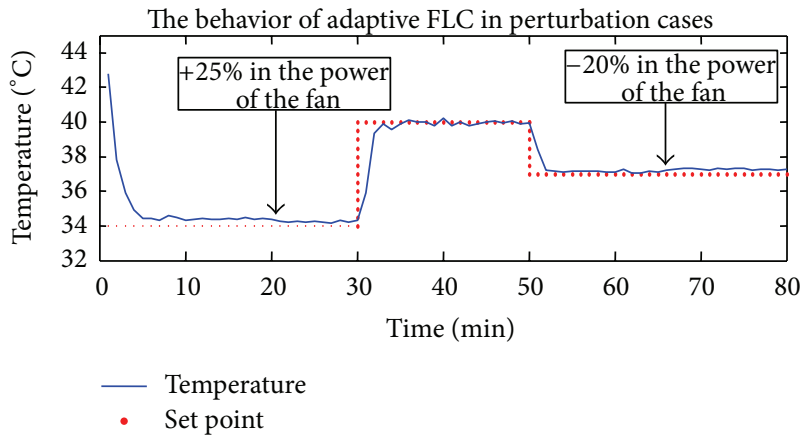

(b)

FIGURE 13: The perturbation effects on the control performance.

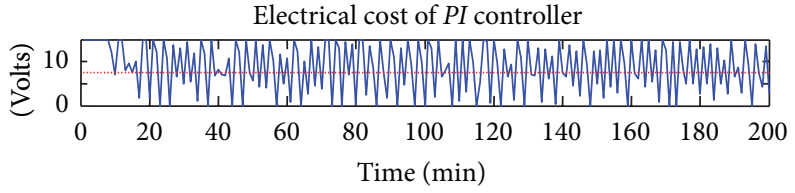

(a)

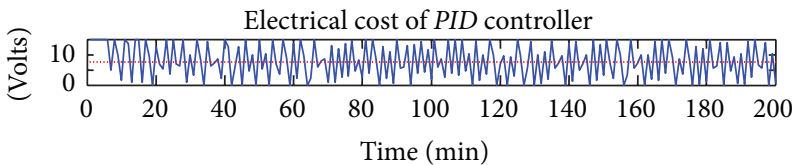

(c)

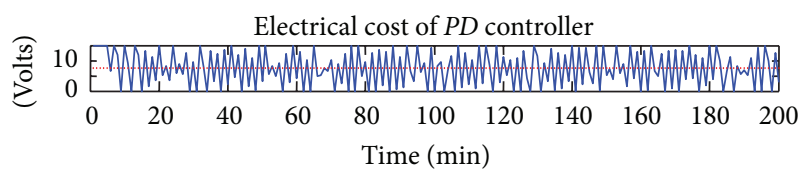

(b)

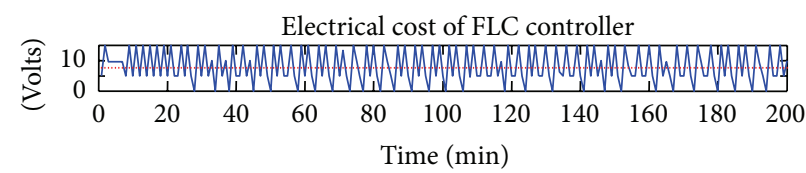

(d)

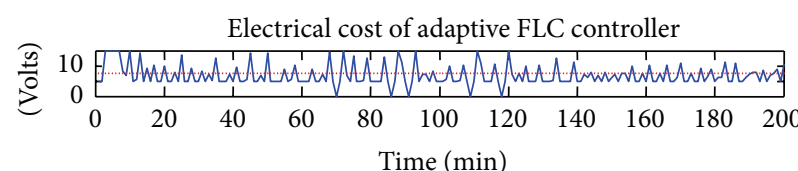

$$
\begin{aligned}
& \text { - The fan feed } \\
& 50 \% \text { of range }
\end{aligned}
$$

(e)

Figure 14: Electrical cost of each used algorithm during 200 minutes.

TABLE 10: Analysis of variance for MSE (sum of squares type III).

\begin{tabular}{lccccc}
\hline Source & $\begin{array}{c}\text { Sum of } \\
\text { squares }\end{array}$ & DF & $\begin{array}{c}\text { Mean } \\
\text { square }\end{array}$ & $F$-ratio & $P$-value \\
\hline Principal effects & & & & & \\
\multicolumn{1}{c}{ A: set points } & 0,069192 & 1 & 0,069192 & 1,98 & 0,1663 \\
B: control & 2,18831 & 4 & 0,547078 & 15,66 & 0,0000 \\
$\begin{array}{l}\text { Residual } \\
\text { Total }\end{array}$ & 1,53669 & 44 & 0,0349247 & & \\
(corrected) & 3,79419 & 49 & & & \\
\hline
\end{tabular}

All the $F$-ratios are based on the residual mean square error.

pass from the rest mode to the maximum of performances which affects the rotor of the fan. Table 7 shows the electric consumption of each algorithm during the simulation time.

Under the same conditions and during the same time of control, the adaptive controller guarantees a very moderate

\begin{tabular}{|c|c|c|c|c|c|}
\hline Source & $\begin{array}{l}\text { Sum of } \\
\text { squares }\end{array}$ & DF & $\begin{array}{c}\text { Mean } \\
\text { square }\end{array}$ & F-ratio & $P$-value \\
\hline \multicolumn{6}{|l|}{ Principal effects } \\
\hline A: set points & 85,2035 & 1 & 85,2035 & 0,04 & 0,8504 \\
\hline B: control & 35365,0 & 4 & 8841,24 & 3,74 & 0,0106 \\
\hline Residual & 104108, & 44 & 2366,09 & & \\
\hline $\begin{array}{l}\text { Total } \\
\text { (corrected) }\end{array}$ & 139558, & 49 & & & \\
\hline
\end{tabular}

TABLE 11: Analysis of variance for EC (sum of squares type III).

All the $F$-ratios are based on the residual mean square error.

electric consumption by generating a feed signal that ensures the smoothness for the fan operation. The remaining controllers have shown a relatively higher consumption, forcing the fan to operate brutally which affects their well-being over the time. 


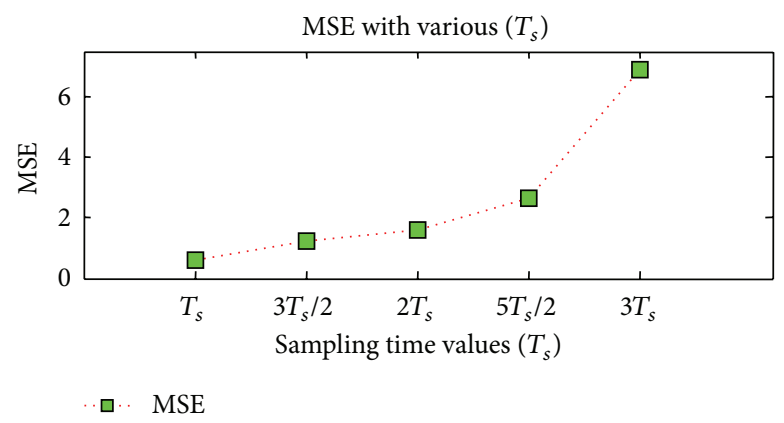

(a)

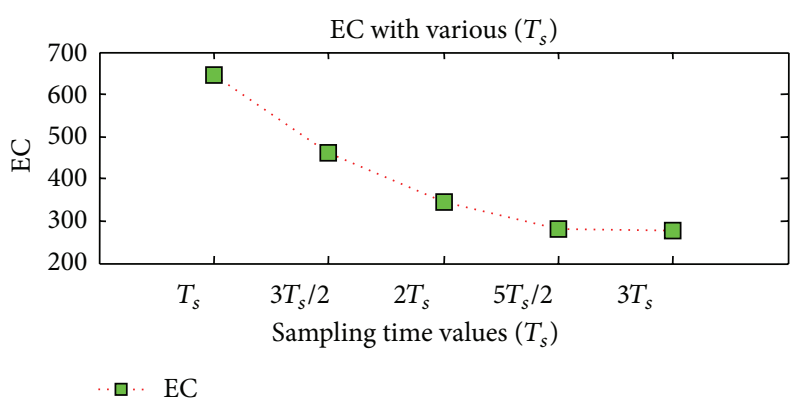

(b)

FIgURE 15: The evolution of MSE and EC using adaptive FLC with various sampling time values.

TABLE 12: Multiple range test for MSE of the Effect B: control.

(a) Method of 95,0\% LSD for MSE of the Effect B: control

\begin{tabular}{lcccc}
\hline Control & Cases & Mean LS & $\sum$ LS & $\begin{array}{c}\text { Homogeneous } \\
\text { groups }\end{array}$ \\
\hline Adapt FLC & 10 & 0,824 & 0,0590971 & $\mathrm{X}$ \\
FLC & 10 & 1,209 & 0,0590971 & $\mathrm{X}$ \\
PID & 10 & 1,275 & 0,0590971 & $\mathrm{X}$ \\
PD & 10 & 1,291 & 0,0590971 & $\mathrm{X}$ \\
PI & 10 & 1,453 & 0,0590971 & $\mathrm{X}$ \\
\hline
\end{tabular}

(b) The contrast between the used Algorithms

\begin{tabular}{lccc}
\hline Contrast & Sig. & Difference & + /- Limits \\
\hline Adapt-FLC & $*$ & $-0,385$ & 0,168437 \\
Adapt-PID & $*$ & $-0,451$ & 0,168437 \\
Adapt-PD & $*$ & $-0,467$ & 0,168437 \\
Adapt-PI & $*$ & $-0,629$ & 0,168437 \\
FLC-PID & & $-0,066$ & 0,168437 \\
FLC-PD & & $-0,082$ & 0,168437 \\
FLC-PI & $*$ & $-0,244$ & 0,168437 \\
PD-PID & & 0,016 & 0,168437 \\
$P I-P I D$ & $*$ & 0,178 & 0,168437 \\
$P D-P I$ & & $-0,162$ & 0,168437 \\
\hline
\end{tabular}

${ }^{*}$ Indicates a significant difference.

4.6. The Effects of Sampling Time on the Electrical Consumption. The results presented earlier show that the adaptive control policy adopted in this paper guarantees a remarkable reduction in the electrical consumption during the control process compared with conventional controllers (under the same conditions). In this paragraph we will try to focus on the effect of the variation of sampling time $\left(T_{\mathrm{s}}\right)$ regarding the evolution of electrical consumption during the control process, and Figure 15 shows the evolution of the EC (electrical consumption) and the MSE (mean squared error) for various sampling time during $30 \mathrm{~min}$ using the adaptive fuzzy controller (for only one set point).

From the graphs presented in Figure 15 we can easily deduce that the augmentation of sampling time implies a
TABLE 13: Multiple range test for EC of the Effect B: control.

(a) Method of 95,0\% LSD for EC of the Effect B: control

\begin{tabular}{lcccc}
\hline Control & Cases & Mean LS & $\sum$ LS & $\begin{array}{c}\text { Homogeneous } \\
\text { groups }\end{array}$ \\
\hline Adapt FLC & 10 & 1059,05 & 15,3821 & $\mathbf{X}$ \\
FLC & 10 & 1128,28 & 15,3821 & $\mathrm{X}$ \\
PID & 10 & 1129,29 & 15,3821 & $\mathrm{X}$ \\
PD & 10 & 1088,19 & 15,3821 & $\mathrm{X}$ \\
$P I$ & 10 & 1110,43 & 15,3821 & $\mathrm{X}$ \\
\hline
\end{tabular}

(b) The contrast between the used Algorithms

\begin{tabular}{lccc}
\hline Contrast & Sig. & Difference & + /- Limits \\
\hline Adapt-FLC & $*$ & $-69,23$ & 43,8415 \\
Adapt-PID & $*$ & $-70,244$ & 43,8415 \\
Adapt-PD & & $-29,143$ & 43,8415 \\
Adapt-PI & $*$ & $-51,38$ & 43,8415 \\
FLC-PID & & $-1,014$ & 43,8415 \\
FLC-PD & & 40,087 & 43,8415 \\
FLC-PI & & 17,85 & 43,8415 \\
PD-PID & & $-41,101$ & 43,8415 \\
PI-PID & & $-18,864$ & 43,8415 \\
PD-PI & & $-22,237$ & 43,8415 \\
\hline
\end{tabular}

${ }^{*}$ Indicates a significant difference.

reduction of electrical consumption with an expansion in the committed error, and it is important to note here that the error growth registered using a slightly larger sampling time $\left(3 T_{\mathrm{s}} / 2\right.$ and $\left.2 T_{\mathrm{s}}\right)$ has little effect on the control performance.

In other words, using an adaptive controller with the usual sampling time provides the best control performance, high precision, and low power consumption (Figure 11 and Table 5) compared to the remaining controllers, but the use of the adaptive controller with a slightly longer sampling time $\left(3 T_{s} / 2\right.$ or $\left.2 T_{s}\right)$ can economize even more this consumption of up to $50 \%$ (Table 8 ). The error growth caused by using a larger sampling time seems like a drawback, and this new error is still within the allowable range, because it is almost 
TABLE 14: The MSE obtained under several type of perturbations.

\begin{tabular}{|c|c|c|c|c|c|c|c|c|c|c|c|c|c|}
\hline \multirow{2}{*}{\multicolumn{2}{|c|}{ Perturbation }} & \multicolumn{6}{|c|}{ Set points 1} & \multicolumn{6}{|c|}{ Set points 2} \\
\hline & & MSE 1 & MSE 2 & MSE 3 & MSE 4 & MSE 5 & $\sum \mathrm{MSE} / 5$ & MSE 1 & MSE 2 & MSE 3 & MSE 4 & MSE 5 & $\sum \mathrm{MSE} / 5$ \\
\hline \multirow{2}{*}{$+30 \%$} & Adap FLC & 1.02 & 0.82 & 0.75 & 0.66 & 0.75 & 0.8 & 1.34 & 1.03 & 0.82 & 1.02 & 0.8 & 1 \\
\hline & FLC & 1.24 & 1.04 & 1.4 & 1.3 & 1.1 & 1.21 & 1.56 & 1.47 & 1.5 & 1.55 & 1.85 & 1.6 \\
\hline \multirow{2}{*}{$+15 \%$} & Adap FLC & 1.18 & 1.03 & 0.78 & 0.93 & 0.78 & 0.94 & 0.95 & 0.93 & 0.95 & 0.92 & 0.81 & 0.91 \\
\hline & FLC & 1.2 & 1.16 & 1.1 & 1.23 & 1.28 & 1.2 & 1.13 & 1.28 & 1.36 & 1.37 & 1.41 & 1.31 \\
\hline \multirow{2}{*}{$-15 \%$} & Adap FLC & 0,92 & 0.91 & 0.97 & 0.94 & 0.9 & 0.92 & 1.05 & 1.07 & 1.07 & 0.95 & 0.98 & 1 \\
\hline & FLC & 1,3 & 1.16 & 1.2 & 1.17 & 1.5 & 1.26 & 1.1 & 1.14 & 1.27 & 1.58 & 1.86 & 1.4 \\
\hline \multirow{2}{*}{$-30 \%$} & Adap FLC & 1.01 & 0.96 & 0.91 & 0.87 & 0.84 & 0.91 & 1.01 & 0.89 & 1.07 & 0.9 & 0.94 & 0.96 \\
\hline & FLC & 3.76 & 2.96 & 3.72 & 3.88 & 2.7 & 3.4 & 1.84 & 1.9 & 1.66 & 1.7 & 1.26 & 1.7 \\
\hline
\end{tabular}

TABLE 15: Analysis of variance for MSE (sum of squares type III).

\begin{tabular}{|c|c|c|c|c|c|}
\hline source & Sum of squares & DF & Mean square & $F$-ratio & $P$-value \\
\hline \multicolumn{6}{|l|}{ Principal efects } \\
\hline A: set points & 0,20402 & 1 & 0,20402 & 0,95 & 0,3341 \\
\hline B: control & 9,63272 & 1 & 9,63272 & 44,63 & 0,0000 \\
\hline C: noise & 5,60313 & 3 & 1,86771 & 8,65 & 0,0001 \\
\hline Residual & 15,9709 & 74 & 0,215823 & & \\
\hline Total (corrected) & 31,4108 & 79 & & & \\
\hline
\end{tabular}

All the $F$-ratios are based on the residual mean square error.

TABLE 16: Multiple range test for MSE of the Effect B: control (perturbation cases).

(a) Method of 95,0\% LSD for MSE of the Effect B: control

\begin{tabular}{lcccc}
\hline Control & Cases & Mean LS & $\sum$ LS & $\begin{array}{c}\text { Homogeneous } \\
\text { groups }\end{array}$ \\
\hline Adapt FLC & 40 & 0,93575 & 0,0734546 & X \\
FLC & 40 & 1,62975 & 0,0734546 & X \\
\hline
\end{tabular}

(b) The contrast between the used Algorithms

\begin{tabular}{lccc}
\hline Contrast & Sig. & Difference & +/- Limits \\
\hline Adapt & $*$ & $-0,694$ & 0,206987 \\
FLC-FLC & $*$ & & \\
\hline
\end{tabular}

${ }^{*}$ Indicates a significant difference.

similar to the error obtained by the conventional controllers with the usual sampling time, that is, in cases where the control process does not require a very high precision (airconditions in building, ext.); the use of our adaptive fuzzy logic controller with a relatively long sampling time will ensure a very important energy savings with a tolerable margin of error. Table 8 presents a real consumption during 30 min using the adaptive FLC.

On the other hand, the controller output is the actuator feeding, and observing the graphs (Figure 14), we can note that the adaptive controller produces a very smooth signal with moderate values without achieving the extremes ranges of the actuator and without providing many changes in the input signal; that is, feeding the actuator with stable and moderate values for a relatively long time guarantees a perfect operation of this latter within their optimum range which will be reflected in the actuator well-being over the time.

4.7. Statistical Analysis of Results. After demonstrating the superiority and the effectiveness of our algorithm compared to the other controllers using a numerical and graphical comparison of the real simulation results, we proceed to illustrate this superiority by making an inferential statistical analysis based on the results obtained by repeating each simulation five times and with two different sets of set points under the same conditions.

This analysis aims to demonstrate the validity and the level of accuracy of our approach in future simulations.

Table 9 presents the results of all realized simulations, giving the MSE and the EC obtained of each algorithm using two sets of set points.

Table 14 shows the results of simulations under different types of disturbance, giving the MSE obtained using FLC and AdaptFLC; each simulation was repeated five times with two different sets of set points.

4.7.1. Analysis of Variance (ANOVA). To analyze the variance of the MSE we proceed to construct the ANOVA table that decomposes the MSE variability in contributions due to several factors. We note here that the sum of squares type III has been chosen (by default); the contribution of each factor is measured by avoiding the effects of the remaining factors.

The $P$ value tests the statistical significance of each factor, and a $P$ value less than 0.05 implies that this factor has a statistically significant effect on the MSE with a $95 \%$ confidence level, so from the data presented in the ANOVA 
table we can determine if the effects have a statistically significant effect on the MSE.

Multiple Range Test for MSE of Control. In the multiple range test a multiple comparison procedure is done to determine which means are significantly different from others, the method used to discriminate between the means is the method of Least Significant Difference (LSD) of Fisher.

The asterisk adjacent to the pairs indicates that these pairs show statistically significant differences with a $95 \%$ confidence level.

Statistical Results. From the ANOVA Tables 10 and 11 we can deduce that the effect $B$ : control has a statistically significant effect on the MSE registered with a 95\% confidence level $\left(P_{B}\right.$ value $<0.05)$; that is, if we repeat the simulations there is a 95\% risk of having similar MSE. On the other hand, from Tables 12(a) and 12(b) and Tables 13(a) and 13(b) of multiple range test of the MSE and the EC we can deduce that there are significant differences (in MSE and in EC) between our adaptive algorithm and the remaining algorithms with a 95\% level of confidence; that is, if we repeat the simulations several times there is a $95 \%$ risk of having similar MSE and similar differences in EC between our algorithm and the others.

For the cases of perturbation, we note that from the ANOVA Table 15 that the effects C: noise and B: control have a statistically significant effect on the MSE registered with a 95\% confidence level (both $P$ value $<0.05$ ); that is, if we repeat the simulations with the same degree of perturbations there is a $95 \%$ risk of having the MSE in the same range. On the other hand, Tables 16(a) and 16(b) of multiple range test of the MSE for CONTROL prove that there is a statistically significant difference (between the MSE registered by our adaptive algorithm and the other algorithms in cases of disturbances) of $95 \%$ level of confidence; that is, if we repeat the simulations several times, there is a $95 \%$ risk of having the same difference of error between our algorithm and the FLC.

\section{Conclusion}

This paper presents a multilevel comparative study between several conventional control algorithms and a self-organized and online adaptive fuzzy logic controller. Using the same training board and under the same conditions, we have tested the algorithms behavior on this plant. Both the conventional fuzzy logic controller and the controllers based on the $P, I$, and $D$ structures have shown their capabilities to control the plant with a reasonable error. The adaptive algorithm without any offline pretraining and starting with no definite rule base has been able to collect a very strong background about the real behavior of the plant in real time, and using this valuable background, the self-organization and the autoLearning will achieve the optimal configuration of the internal parameters of the adaptive controller (suitable rules consequents and optimal position for member ship functions) and improve the committed error during the control process. The adaptive algorithm does not need any complex mathematical models. It only needs very limited information from the plant. The monotonicity and the delay of the plant were the only information used by the adaptive algorithm to optimize the error at the plant output. Another advantage of this algorithm in addition to its precision and its ease of implementation is the energy saving. The adaptive controller reaches to control the process very accurately with great smoothness and low power consumption. The results comparison can prove the superiority of the proposed self-organized and online adaptive FLC against the remaining algorithms and the statistical study can prove the validity of this technique.

\section{References}

[1] E. H. Mamdani and S. Assilian, "Experiment in linguistic synthesis with a fuzzy logic controller," International Journal of Man-Machine Studies, vol. 7, no. 1, pp. 1-13, 1975.

[2] T. J. Procyk and E. H. Mamdani, "A linguistic self-organizing process controller," Automatica, vol. 15, no. 1, pp. 15-30, 1979.

[3] Y.-C. Hsueh and S.-F. Su, "Fuzzy sliding controller design with adaptive approximate error feedback," International Journal of Fuzzy Systems, vol. 11, no. 1, pp. 36-43, 2009.

[4] Y. Pan, M. J. Er, D. Huang, and Q. Wang, "Adaptive fuzzy control with guaranteed convergence of optimal approximation error," IEEE Transactions on Fuzzy Systems, vol. 19, no. 5, pp. 807-818, 2011.

[5] C.-F. Hsu, C.-M. Lin, and M.-C. Li, "Adaptive dynamic RBF fuzzy neural controller design with a constructive learning," International Journal of Fuzzy Systems, vol. 13, no. 3, pp. 175-184, 2011.

[6] Q. Zhou, P. Shi, J. Lu, and S. Xu, "Adaptive output-feedback fuzzy tracking control for a class of nonlinear systems," IEEE Transactions on Fuzzy Systems, vol. 19, no. 5, pp. 972-982, 2011.

[7] M. Nie and W. W. Tan, "Stable adaptive fuzzy PD plus PI controller for nonlinear uncertain systems," Fuzzy Sets and Systems, vol. 179, no. 1, pp. 1-19, 2011.

[8] H. Chaoui and P. Sicard, "Adaptive fuzzy logic control of permanent magnet synchronous machines with nonlinear friction," IEEE Transactions on Industrial Electronics, vol. 59, no. 2, pp. 1123-1133, 2012.

[9] T. Guo and G. Liu, "Adaptive fuzzy control for unknown nonlinear time-delay systems with virtual control functions," International Journal of Control, Automation and Systems, vol. 9, no. 6, pp. 1227-1234, 2011.

[10] R.-J. Lian, "Design of an enhanced adaptive self-organizing fuzzy sliding-mode controller for robotic systems," Expert Systems with Applications, vol. 39, no. 1, pp. 1545-1554, 2012.

[11] H.-C. Lu, M.-H. Chang, and C.-H. Tsai, "Adaptive selfconstructing fuzzy neural network controller for hardware implementation of an inverted pendulum system," Applied Soft Computing Journal, vol. 11, no. 5, pp. 3962-3975, 2011.

[12] C.-H. Wang, C.-H. Lin, B.-K. Lee, C.-N. Jimmy Liu, and C. Su, "Adaptive two-stage fuzzy temperature control for an electroheat system," International Journal of Fuzzy Systems, vol. 11, no. 1, pp. 59-66, 2009.

[13] T. O. Kowalska, M. Dybkowski, and K. Szabat, "Adaptive sliding-mode neuro-fuzzy control of the two-mass induction motor drive without mechanical sensors," IEEE Transactions on Industrial Electronics, vol. 57, no. 2, pp. 553-564, 2010.

[14] A. Fadaei and K. Salahshoor, "A novel real-time fuzzy adaptive auto-tuning scheme for cascade PID controllers," International Journal of Control, Automation and Systems, vol. 9, no. 5, pp. 823-833, 2011. 
[15] M. J. Er, F. Liu, and M. B. Li, "Self-constructing fuzzy neural networks with extended Kalman filter," International Journal of Fuzzy Systems, vol. 12, no. 1, pp. 66-72, 2010.

[16] S. Cho, J. Kim, and S.-T. Chung, "Self-organizing fuzzy controller based on fuzzy neural network," Advances in Soft Computing, vol. 41, pp. 185-194, 2007.

[17] R.-J. Lian, B.-F. Lin, and W.-T. Sie, "Self-organizing fuzzy control of active suspension systems," International Journal of Systems Science, vol. 36, no. 3, pp. 119-135, 2005.

[18] C.-J. Wu, C.-N. Ko, Y.-Y. Fu, and C.-H. Tseng, "A genetic-based design of auto-tuning fuzzy PID controllers," International Journal of Fuzzy Systems, vol. 11, no. 1, pp. 49-58, 2009.

[19] R.-J. Lian, "Intelligent controller for robotic motion control," IEEE Transactions on Industrial Electronics, vol. 58, no. 11, pp. 5220-5230, 2011

[20] P. A. Phan and T. J. Gale, "Direct adaptive fuzzy control with a self-structuring algorithm," Fuzzy Sets and Systems, vol. 159, no. 8, pp. 871-899, 2008.

[21] J. Pargfrieder and H. P. Jörgl, "An integrated control system for optimizing the energy consumption and user comfort in buildings, Computer Aided Control System Design," IEEE International Symposium on Aided Control System Design, vol. 127, 2002.

[22] C. B. Chiou, C. H. Chiou, C. M. Chu, and S. L. Lin, "The application of fuzzy control on energy saving for multi-unit room air-conditioners," Applied Thermal Engineering, vol. 29, no. 2-3, pp. 310-316, 2009.

[23] R. Talebi-Daryani and J. Pfaff, "Intelligent control and power management of air conditioning systems using fuzzy logic and local oeration networks," in Proceedings of the 5th Biannual World Automation Congress, vol. 14, pp. 195-202, June 2002.

[24] Y.-W. Bai and C.-H. Cheng, "Using an embedded controller with fuzzy logic to reduce power consumption of mobile computers," in Proceedings of the 36th Annual Conference of the IEEE Industrial Electronics Society (IECON '10), pp. 236-241, November 2010.

[25] J. G. Ziegler and N. B. Nichols, "Optimum settings for automatic controllers," Transactions of ASME, vol. 64, pp. 759-768, 1942.

[26] G.-R. Yu and C.-S. You, "Nonlinear robust control of fuzzy timedelay systems," International Journal of Fuzzy Systems, vol. 11, no. 1, pp. 29-35, 2009.

[27] C. Peng, L.-Y. Wen, and J.-Q. Yang, "On delay-dependent robust stability criteria for uncertain T-S fuzzy systems with interval time-varying delay," International Journal of Fuzzy Systems, vol. 13, no. 1, pp. 35-44, 2011.

[28] A. Boulkroune, M. M'Saad, and M. Farza, "Adaptive fuzzy controller for multivariable nonlinear state time-varying delay systems subject to input nonlinearities," Fuzzy Sets and Systems, vol. 164, no. 1, pp. 45-65, 2011.

[29] R. -J. Lian, "Enhanced adaptive self-organizing fuzzy slidingmode controller for active suspension systems," IEEE Transactions on Industrial Electronics, vol. 60, no. 3, pp. 958-968, 2013.

[30] H. T. Do and K. K. Ahn, "Velocity control of a secondary controlled closed-loop hydrostatic transmission system using an adaptive fuzzy sliding mode controller," Journal of Mechanical Science and Technology, vol. 27, no. 3, pp. 875-884, 2013.

[31] A. M. Abusorrah, "Optimal power flow using adaptive fuzzy logic controllers," Mathematical Problems in Engineering, vol. 2013, Article ID 975170, 7 pages, 2013.

[32] C.-H. Kao, C.-F. Hsu, and H.-S. Don, "Design of an adaptive self-organizing fuzzy neural network controller for uncertain nonlinear chaotic systems," Neural Computing and Applications, vol. 6, no. 21, pp. 1243-1253, 2011.

[33] I. Rojas, H. Pomares, J. Gonzalez et al., "Adaptive fuzzy controller: application to the control of the temperature of a dynamic room in real time," Fuzzy Sets and Systems, vol. 157, no. 16, pp. 2241-2258, 2006. 

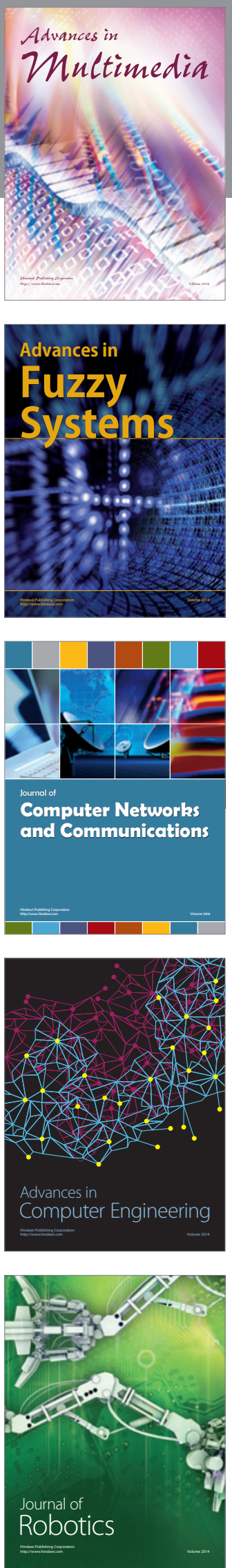

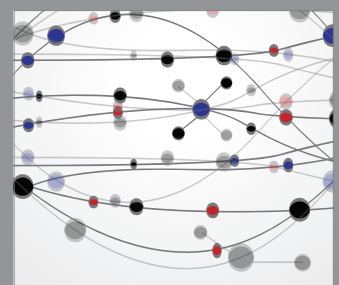

The Scientific World Journal
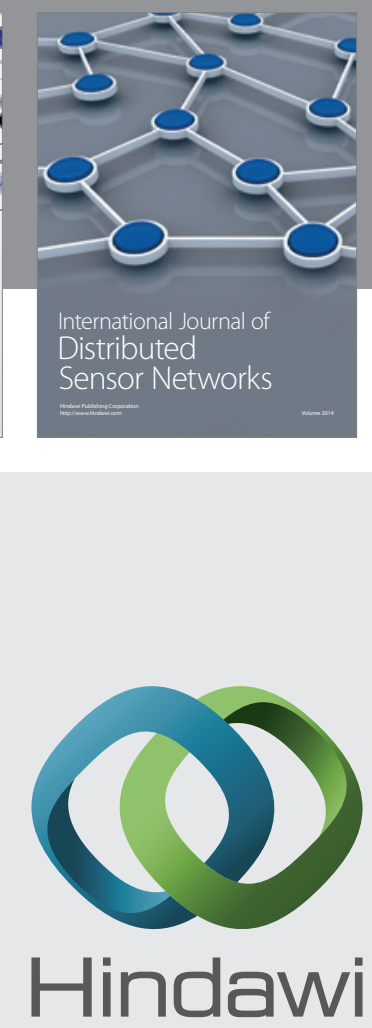

Submit your manuscripts at

http://www.hindawi.com
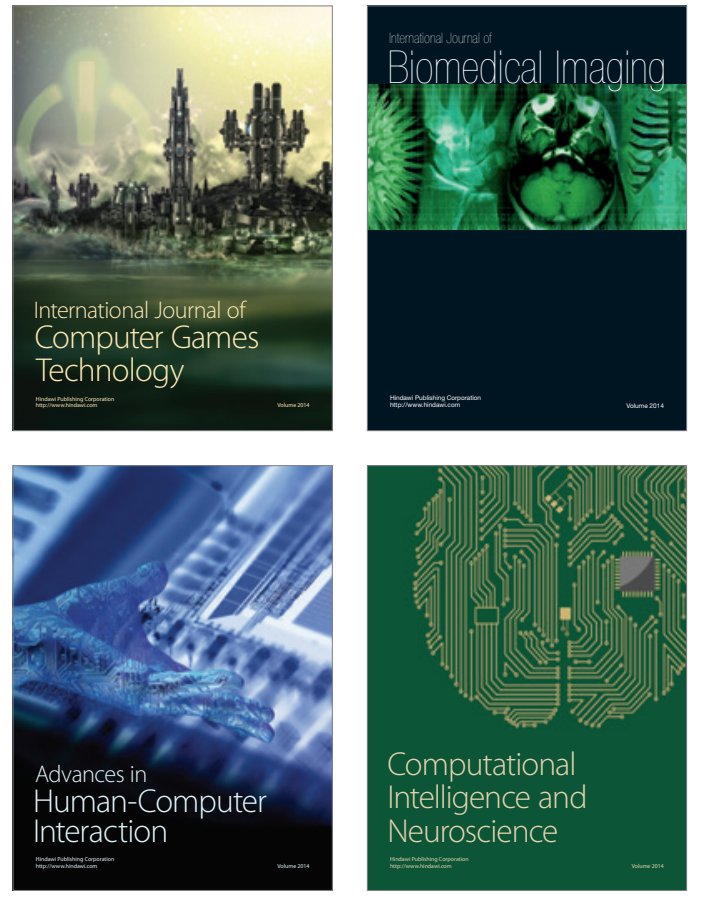
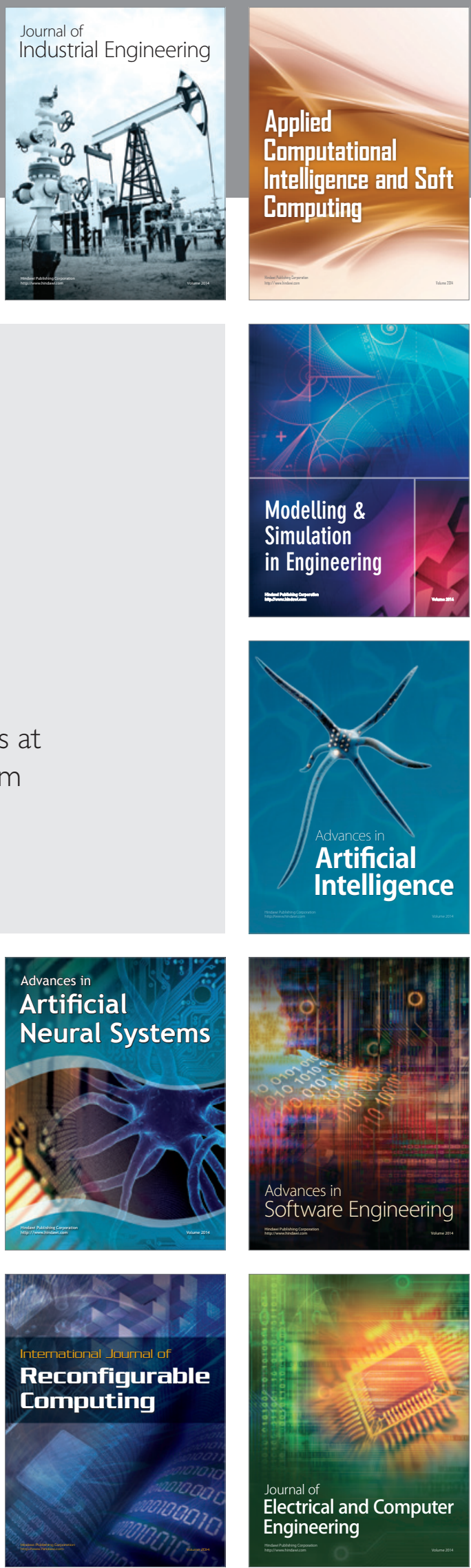